\title{
Stability of Hyperbolic Equilibrium Solution of Second Order Nonlinear Rational Difference Equation
}

\author{
S. Atawna, ${ }^{1}$ R. Abu-Saris, ${ }^{2}$ E. S. Ismail, ${ }^{1}$ and I. Hashim ${ }^{1}$ \\ ${ }^{1}$ School of Mathematical Sciences, Universiti Kebangsaan Malaysia, 43600 Bangi, Selangor, Malaysia \\ ${ }^{2}$ Department of Basic Sciences, King Saud bin Abdulaziz University for Health Sciences, Riyadh 11426, Saudi Arabia
}

Correspondence should be addressed to S. Atawna; s_atawna@yahoo.com

Received 4 January 2015; Accepted 25 March 2015

Academic Editor: Richard Saurel

Copyright (C) 2015 S. Atawna et al. This is an open access article distributed under the Creative Commons Attribution License, which permits unrestricted use, distribution, and reproduction in any medium, provided the original work is properly cited.

Our goal in this paper is to investigate the global asymptotic stability of the hyperbolic equilibrium solution of the second order rational difference equation $x_{n+1}=\left(\alpha+\beta x_{n}+\gamma x_{n-1}\right) /\left(A+B x_{n}+C x_{n-1}\right), n=0,1,2, \ldots$, where the parameters $A \geq 0$ and $B, C, \alpha, \beta, \gamma$ are positive real numbers and the initial conditions $x_{-1}, x_{0}$ are nonnegative real numbers such that $A+B x_{0}+C x_{-1}>0$. In particular, we solve Conjecture 5.201.1 proposed by Camouzis and Ladas in their book (2008) which appeared previously in Conjecture 11.4.2 in Kulenović and Ladas monograph (2002).

\section{Introduction}

Rational difference equations, particularly bilinear ones, that is,

$$
x_{n+1}=\frac{\alpha+\sum_{i=0}^{k} \alpha_{i} x_{n-i}}{\beta+\sum_{j=0}^{\ell} \beta_{j} x_{n-j}} ; \quad n=0,1,2,3, \ldots
$$

attracted the attention of many researchers recently. For example, see the articles [1-9]. As it turns out, many models, such as population models in mathematical biology, are members of the family of rational difference equations. The behavior of solutions of rational difference equations can provide prototypes towards the development of the basic theory of the global behavior of solutions of nonlinear difference equations of order greater than one. Hence, the study of this family of equations is important from both a theoretical point of view and the point of view of applications.

For the general theory of difference equations, one can refer to Agarwal [10], Elaydi [11], Kelley and peterson [12], and the monograph of Kocic and Ladas [13]. Many rational difference equations were studied extensively in $[14,15]$ and the references cited therein.
Asystematic study of the second order rational difference equation of the form

$$
x_{n+1}=\frac{\alpha+\beta x_{n}+\gamma x_{n-1}}{A+B x_{n}+C x_{n-1}}, \quad n=0,1,2, \ldots,
$$

where the parameters $\alpha, \beta, \gamma, A, B, C$ and the initial conditions $x_{-1}, x_{0}$ are nonnegative real numbers, was considered in the Kulenović and Ladas monograph [15]. They came up with the idea of setting one or more parameters in (2) to zero and studying the resulting equation with fewer parameters. This approach gives rise to 49 different cases which exhibit variety dynamics. They presented the known results such as [16-23]. Next, Kulenović and Ladas [15] derived several ones on the boundedness, the global stability, and the periodicity of solutions of all rational difference equations of the form (2). Furthermore, they posed several open problems and conjectures related to this equation and its functional generalization.

Even after a sustained effort by many researchers such as [24-28], there were some cases of the 49 different cases that have not been investigated till 2007. Amleh et al. in $[29,30]$ give an up-to-date account on recent developments related to (2) up to 2007. Furthermore, they reposed several open problems and conjectures related to this equation. 
Camouzis and Ladas in [14] summarize the progress up to 2008 in the study of the 49 cases of (2) as subcases of the 255 special cases of the more general third-order rational difference equation

$$
x_{n+1}=\frac{\alpha+\beta x_{n}+\gamma x_{n-1}+\delta x_{n-2}}{A+B x_{n}+C x_{n-1}+D_{x_{n-2}}}, \quad n=0,1,2, \ldots,
$$

where the parameters $\alpha, \beta, \gamma, \delta, A, B, C, D$ and the initial conditions $x_{-2}, x_{-1}, x_{0}$ are nonnegative real numbers. In their book, Camouzis and Ladas [14] have posed a series of open problems and conjectures related to (3). In addition, they reposed open problems and conjectures on these remaining equations of (2) that have resisted analysis so far.

Recently, the work done by many researchers such as [31-39] have solved many open problems and conjectures proposed in $[14,15,29,30]$ related to (2) and have led to the development of some general theory about difference equation. However, as confirmed by Professor Kulenović (personal communication, August 24, 2014), the case $A=0$ remains open.

Our approach handles the aforementioned case as well as other cases. Furthermore, the results of this paper improve and extend the asymptotic results in [15, Chapter 11]. Indeed, our results provide affirmative answer to the following conjecture proposed by Camouzis and Ladas in [14, Conjecture 5.201.1].

Conjecture 1. This shows that, for the equilibrium $\bar{x}$ of (2),

Local Asymptotic Stability $\Longrightarrow$ Global Attractivity. (4)

It is worth mentioning that the aforementioned conjecture appeared previously in the Kulenovic and Ladas monograph [15, Conjecture 11.4.2].

To this end and using the transformation

$$
x_{n}=\frac{\gamma}{C} y_{n}
$$

equation (2) reduces to

$$
y_{n+1}=\frac{r+p y_{n}+y_{n-1}}{z+q y_{n}+y_{n-1}}, \quad n=0,1,2, \ldots,
$$

where

$$
r=\frac{\alpha C}{\gamma^{2}}, \quad p=\frac{\beta}{\gamma}, \quad z=\frac{A}{\gamma}, \quad q=\frac{B}{C}
$$

are positive real numbers and the initial conditions $y_{-1}, y_{0}$ are nonnegative real numbers.

The periodic character of positive solutions of (6) has been investigated by the authors in [40]. They showed that the period-two solution is locally asymptotically stable if it exists.

Our results, together with the established results in $[15$, 40 ], give a complete picture of the nature of solutions of the second order rational difference equation of the form (2). We believe that our results are important stepping stone in understanding the behavior of solutions of rational difference equations which provides prototypes towards the development of the basic theory of the global behavior of solutions of nonlinear difference equations of higher order.

That being said, the remainder of this paper is organized as follows. In the next section, a brief description of some definitions and results from the literature that are needed to prove the main results in this paper is given. It is worth mentioning that there are few global attractivity results in the literature that can be applied to rational difference equations of the form (2). Next we establish our main results in Sections 3-5. We determine the local stability character of (2) in Section 3. Section 4 examines the existence of intervals which attract all solutions of (2). In Section 5, we investigate the global asymptotic stability of the hyperbolic equilibrium solution of (6). Next, in Section 6 we consider several numerical examples generated by MATLAB to illustrate the results of the previous sections and to support our theoretical discussion. Finally, we conclude in Section 7 with suggestion for future research.

\section{Preliminaries}

For the sake of self-containment and convenience, we recall the following definitions and results from [15].

Let $I$ be a nondegenerate interval of real numbers and let $f: I \times I \rightarrow I$ be a continuously differentiable function. Then for every set of initial conditions $x_{0}, x_{-1} \in I$, the difference equation

$$
x_{n+1}=f\left(x_{n}, x_{n-1}\right), \quad n=0,1, \ldots
$$

has a unique solution $\left\{x_{n}\right\}_{n=-1}^{\infty}$.

A constant sequence, $x_{n}=\bar{x}$ for all $n$ where $\bar{x} \in I$, is called an equilibrium solution of (8) if

$$
\bar{x}=f(\bar{x}, \bar{x}) .
$$

Definition 2. Let $\bar{x}$ be an equilibrium solution of (8).

(i) $\bar{x}$ is called locally stable if, for every $\epsilon>0$, there exists $\delta>0$ such that, for all $x_{0}, x_{-1} \in I$, with $\left|x_{0}-\bar{x}\right|+$ $\left|x_{-1}-\bar{x}\right|<\delta$, we have

$$
\left|x_{n}-\bar{x}\right|<\epsilon \quad \forall n \geq-1 .
$$

(ii) $\bar{x}$ is called locally asymptotically stable if it is locally stable and if there exists $\gamma>0$, such that, for all $x_{0}, x_{-1} \in I$, with $\left|x_{0}-\bar{x}\right|+\left|x_{-1}-\bar{x}\right|<\gamma$, we have

$$
\lim _{n \rightarrow \infty} x_{n}=\bar{x}
$$

(iii) $\bar{x}$ is called a global attractor if, for every $x_{0}, x_{-1} \in I$, we have

$$
\lim _{n \rightarrow \infty} x_{n}=\bar{x}
$$

(iv) $\bar{x}$ is called globally asymptotically stable if it is locally stable and a global attractor.

(v) $\bar{x}$ is called unstable if it is not stable. 
(vi) $\bar{x}$ is called a source, or a repeller, if there exists $r>0$ such that, for all $x_{0}, x_{-1} \in I$, with $0<\left|x_{0}-\bar{x}\right|+\mid x_{-1}-$ $\bar{x} \mid<r$, there exists $N \geq 1$ such that

$$
\left|x_{N}-\bar{x}\right| \geq r
$$

Clearly a source is an unstable equilibrium.

Definition 3. Let

$$
a=\frac{\partial f}{\partial u}(\bar{x}, \bar{x}), \quad b=\frac{\partial f}{\partial v}(\bar{x}, \bar{x})
$$

denote the partial derivatives of $f(u, v)$ evaluated at the equilibrium $\bar{x}$ of (8). Then the equation

$$
y_{n+1}=a y_{n}+b y_{n-1}, \quad n=0,1, \ldots
$$

is called the linearized equation associated with (8) about the equilibrium solution $\bar{x}$.

Theorem 4 (linearized stability). (a) If both roots of the quadratic equation

$$
\lambda^{2}-a \lambda-b=0
$$

lie in the open unit disk $|\lambda|<1$, then the equilibrium $\bar{x}$ of (8) is locally asymptotically stable.

(b) If at least one of the roots of (16) has absolute value greater than one, then the equilibrium $\bar{x}$ of (8) is unstable.

(c) A necessary and sufficient condition for both roots of (16) to lie in the open unit disk $|\lambda|<1$ is

$$
|a|<1-b<2 \text {. }
$$

In this case the locally asymptotically stable equilibrium $\bar{x}$ is also called a sink.

(d) A necessary and sufficient condition for both roots of (16) to have absolute value greater than one is

$$
|b|>1, \quad|a|<|1-b| .
$$

In this case $\bar{x}$ is a repeller.

(e) A necessary and sufficient condition for one root of (16) to have absolute value greater than one and for the other to have absolute value less than one is

$$
a^{2}+4 b>0, \quad|a|>|1-b| .
$$

In this case the unstable equilibrium $\bar{x}$ is called a saddle point.

( $f$ ) A necessary and sufficient condition for a root of (16) to have absolute value equal to one is

$$
|a|=|1-b|
$$

or

$$
b=-1, \quad|a| \leq 2 .
$$

Theorem 5. Consider the difference equation (8). Let $I=[a, b]$ be some interval of real numbers and assume that

$$
f:[a, b] \times[a, b] \longrightarrow[a, b]
$$

is a continuous function satisfying the following properties.

(a) $f(x, y)$ is nonincreasing in $x \in[a, b]$ for each $y \in$ $[a, b]$, and $f(x, y)$ is nondecreasing in $y \in[a, b]$ for each $x \in[a, b]$;

(b) the difference equation (8) has no solutions of prime period two in $[a, b]$;

then (8) has a unique equilibrium $\bar{x} \in[a, b]$ and every solution of (8) converges to $\bar{x}$.

Theorem 6. Let $I=[a, b]$ be an interval of real numbers and assume that

$$
f:[a, b] \times[a, b] \longrightarrow[a, b]
$$

is a continuous function satisfying the following properties.

(a) $f(x, y)$ is nondecreasing in $x \in[a, b]$ for each $y \in$ $[a, b]$, and $f(x, y)$ is nonincreasing in $y \in[a, b]$ for each $x \in[a, b]$;

(b) if $(m, M) \in[a, b] \times[a, b]$ is a solution of the system

$$
f(m, M)=m, \quad f(M, m)=M
$$

then $m=M$.

Then (8) has a unique equilibrium $\bar{x} \in[a, b]$ and every solution of $(8)$ converges to $\bar{x}$.

Theorem 7. Let $I=[a, b]$ be an interval of real numbers and assume that

$$
f:[a, b] \times[a, b] \longrightarrow[a, b]
$$

is a continuous function satisfying the following properties.

(a) $f(x, y)$ is nonincreasing in each of its arguments;

(b) if $(m, M) \in[a, b] \times[a, b]$ is a solution of the system

$$
f(m, m)=M, \quad f(M, M)=m,
$$

then $m=M$.

Then (8) has a unique equilibrium $\bar{x} \in[a, b]$ and every solution of (8) converges to $\bar{x}$.

Theorem 8. Let $I=[a, b]$ be an interval of real numbers and assume that

$$
f:[a, b] \times[a, b] \longrightarrow[a, b]
$$

is a continuous function satisfying the following properties.

(a) $f(x, y)$ is nondecreasing in each of its arguments;

(b) the equation

$$
f(x, x)=x
$$

has a unique positive solution. 
Then (8) has a unique equilibrium $\bar{x} \in[a, b]$ and every solution of (8) converges to $\bar{x}$.

The following result from [40] will be useful in the sequel.

Theorem 9. (a) When

$$
p+z \geq 1
$$

equation (6) has no nonnegative prime period-two solution.

(b) When

$$
p+z<1
$$

equation (6) has prime period-two solution,

$$
\ldots, \phi, \psi, \phi, \psi, \ldots,
$$

if and only if condition

$$
r<\frac{(1-p-z)[q(1-p-z)-(1+3 p-z)]}{4},
$$

where $\phi$ and $\psi$ are the positive and distinct solutions of the quadratic equation

$$
t^{2}-(1-z-p) t+\frac{p(1-z-p)+r}{q-1}=0, \quad q>1 .
$$

\section{Local Stability}

In this section, we address the local stability of the equilibrium $\bar{y}$ of (6) when all parameters are positive. In particular, we give explicit conditions on the parameter values of (6) for the equilibrium $\bar{y}$ to be locally asymptotically stable.

Equation (6) has a unique positive equilibrium given by

$$
\bar{y}=\frac{1+p-z+\sqrt{(1+p-z)^{2}+4 r(q+1)}}{2(q+1)} .
$$

The linearized equation associated with (6) about the equilibrium solution is given by

$$
z_{n+1}=\frac{p-q \bar{y}}{z+(q+1) \bar{y}} z_{n}+\frac{1-\bar{y}}{z+(q+1) \bar{y}} z_{n-1} .
$$

Therefore, its characteristic equation is

$$
\lambda^{2}-\frac{p-q \bar{y}}{z+(q+1) \bar{y}} \lambda-\frac{1-\bar{y}}{z+(q+1) \bar{y}}=0 .
$$

By applying linearized stability (Theorem 4(c)) we have the following result.

Theorem 10. (a) Assume that

$$
p+z \geq 1
$$

then the positive equilibrium of (6) is locally asymptotically stable.

\section{(b) Assume that}

$$
p+z<1
$$

then the positive equilibrium of (6) is locally asymptotically stable if and only if

$$
r>\frac{(1-p-z)[q(1-p-z)-(1+3 p-z)]}{4} .
$$

Proof. By employing linearized stability (Theorem 4(c)) we see that condition (17) is equivalent to the following three inequalities:

$$
\begin{gathered}
\frac{p-q \bar{y}}{z+(q+1) \bar{y}}<1-\frac{1-\bar{y}}{z+(q+1) \bar{y}} \\
\frac{p-q \bar{y}}{z+(q+1) \bar{y}}>\frac{1-\bar{y}}{z+(q+1) \bar{y}}-1 \\
\frac{1-\bar{y}}{z+(q+1) \bar{y}}>-1 .
\end{gathered}
$$

Inequality (40) is satisfied if and only if $p-z+1<$ $2(q+1) \bar{y}$, which is always satisfied by (34). Inequality (42) is satisfied if and only if $-1-z<q \bar{y}$, which is always satisfied since $z, q, \bar{y}$ are positive.

Inequality (41) is satisfied if and only if $1-p-z<2 \bar{y}$. Hence $\bar{y}$ is locally asymptotically stable (sink) if and only if

$$
1-p-z<2 \bar{y}
$$

Clearly the equilibrium $\bar{y}$ is the positive solution of the quadratic equation

$$
(q+1) \bar{y}^{2}+(z-p-1) \bar{y}-r=0 .
$$

Now set

$$
F(u)=(q+1) u^{2}+(z-p-1) u-r
$$

then Inequality (43) is satisfied if and only if either

$$
p+z \geq 1
$$

or

$$
p+z<1, \quad F\left(\frac{1-p-z}{2}\right)<0
$$

that is,

$$
\begin{aligned}
(q+1) & \left(\frac{1-p-z}{2}\right)^{2}+(z-p-1)\left(\frac{1-p-z}{2}\right)-r<0, \\
\Longleftrightarrow & (q+1)(1-p-z)^{2}+2(z-p-1)(1-p-z) \\
& \quad-4 r<0, \\
\Longleftrightarrow & (1-p-z)[(q+1)(1-p-z)+2(z-p-1)] \\
& \quad-4 r<0, \\
\Longleftrightarrow & (1-p-z)[q(1-p-z)-(1+3 p-z)] \\
& -4 r<0,
\end{aligned}
$$

from which (39) follows.

The proof is complete. 
TABLE 1: Signs of $\partial f / \partial y_{n}$ and $\partial f / \partial y_{n-1}$ of $(6)$ when $p<q$.

\begin{tabular}{ccccc}
\hline Case & $p z-q r$ & $r-z$ & $\frac{\partial f\left(y_{n}, y_{n-1}\right)}{\partial y_{n}}=\frac{q-p}{\left(z+q y_{n}+y_{n-1}\right)^{2}}\left[\frac{p z-q r}{q-p}-y_{n-1}\right]$ & $\frac{\partial f\left(y_{n}, y_{n-1}\right)}{\partial y_{n-1}}=\frac{q-p}{\left(z+q y_{n}+y_{n-1}\right)^{2}}\left[y_{n}-\frac{r-z}{q-p}\right]$ \\
\hline 1 & + & - & - if $y_{n-1}>\frac{p z-q r}{q-p}$ and + if $y_{n-1}<\frac{p z-q r}{q-p}$ & + \\
2 & 0 & - & - & - \\
3 & - & - & - & + if $y_{n}>\frac{r-z}{q-p}$ and - if $y_{n}<\frac{r-z}{q-p}$ \\
4 & - & 0 & - & +
\end{tabular}

\section{Invariant Intervals}

In this section, we investigate the invariant intervals for (6) in order to obtain convergence results for the solutions of (6).

Let $\left\{y_{n}\right\}_{n=-1}^{\infty}$ be a positive solution of (6). Then we have the following identities:

$$
\begin{gathered}
y_{n+1}-1=\frac{r-z+(p-q) y_{n}}{z+q y_{n}+y_{n-1}}, \quad \text { for } n \geq 0 ; \\
y_{n+1}-\frac{p}{q}=\frac{q r-p z+(q-p) y_{n-1}}{q\left(z+q y_{n}+y_{n-1}\right)}, \quad \text { for } n \geq 0 .
\end{gathered}
$$

We consider the cases where $p<q, p>q$, and $p=q$.

4.1. Case 1: $p<q$. When $p<q$, Identities (49) and (50) are equivalent to the following:

$$
\begin{array}{r}
y_{n+1}-1=\frac{q-p}{z+q y_{n}+y_{n-1}}\left[\frac{r-z}{q-p}-y_{n}\right], \quad \text { for } n \geq 0 \\
y_{n+1}-\frac{p}{q}=\frac{q-p}{q\left(z+q y_{n}+y_{n-1}\right)}\left[y_{n-1}-\frac{p z-q r}{q-p}\right], \\
\text { for } n \geq 0 .
\end{array}
$$

The following remark is straightforward from Identities (51) and (52).

Remark 11. Let $\left\{y_{n}\right\}_{n=-1}^{\infty}$ be a positive solution of (6). Then the following statements are true when $p<q$.

(1) $y_{n+1}>1$ if and only if $y_{n}<(r-z) /(q-p)$.

(2) $y_{n+1}<p / q$ if and only if $y_{n-1}<(p z-q r) /(q-p)$.

Table 1 gives the signs of $\partial f\left(y_{n}, y_{n-1}\right) / \partial y_{n}$ and $\partial f\left(y_{n}\right.$, $\left.y_{n-1}\right) / \partial y_{n-1}$ of (6) in all possible nondegenerate cases when $p<q$.

Lemma 12. Assume that $p<q$ and $r \leq z$.

(1) If $(p z-q r) /(q-p) \leq p / q$ then $\bar{y} \geq p / q$.

(2) If $(p z-q r) /(q-p)>p / q$ then $\bar{y}<p / q$.
Proof. We will prove (1); the proof of (2) is similar and will be omitted. By (34), making use of $(p z-q r) /(q-p) \leq p / q \Rightarrow$ $r \geq\left(p q z-p q+p^{2}\right) / q^{2}, \bar{y}$ is such that

$$
\begin{aligned}
& \bar{y} \geq\left(1+p-z+\frac{1}{q}\right. \\
&\left.\cdot \sqrt{q^{2}(1+p-z)^{2}+4\left(p q z-p q+p^{2}\right)(q+1)}\right) \\
& \cdot(2(q+1))^{-1} .
\end{aligned}
$$

After some elementary algebraic manipulations, Inequality (53) is equivalent to

$$
\begin{aligned}
\bar{y} \geq & (q(1+p-z) \\
& \left.\quad+\sqrt{q^{2}(p+z-1)^{2}+4 p^{2}+4 p q(p+z-1)}\right) \\
& \cdot(2 q(q+1))^{-1} \\
\geq & \frac{q(1+p-z)+\sqrt{(q(p+z-1)+2 p)^{2}}}{2 q(q+1)} \\
\geq & \frac{q(1+p-z)+q(p+z-1)+2 p}{2 q(q+1)}=\frac{p}{q} .
\end{aligned}
$$

The proof is complete.

By Remark 11, Lemma 12, and Table 1, we obtain the following key result.

Theorem 13. Assume that $p<q$ and $r \leq z$; then we have two cases to be considered.

(1) If $p z-q r \leq 0$, then $[p / q, 1]$ is invariant.

(2) If $p z-q r>0$, then we have three subcases to be considered.

(a) If $(p z-q r) /(q-p)<p / q$, then every positive solution of (6) eventually enters and remains in the interval $[p / q, 1]$. 


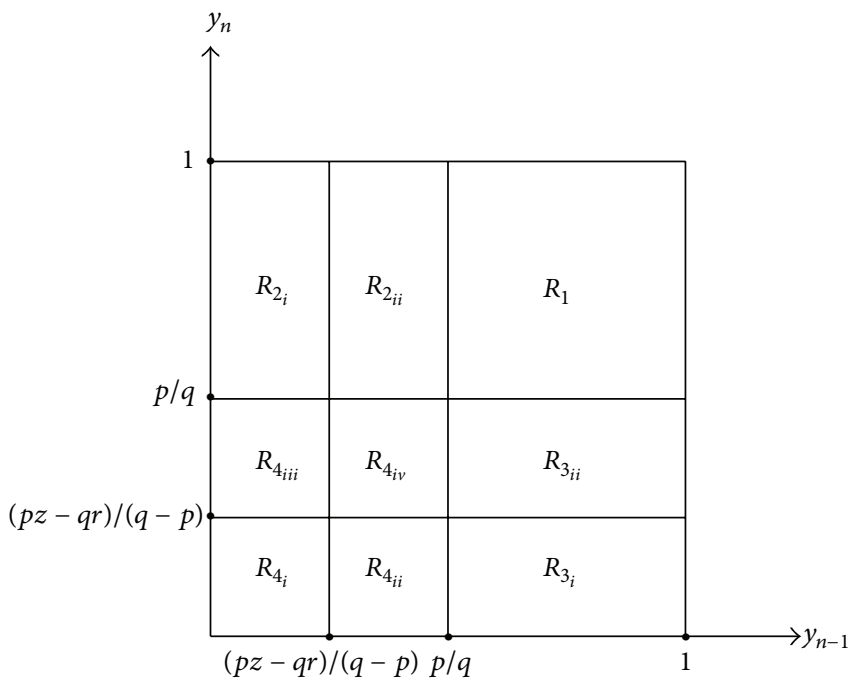

(a)

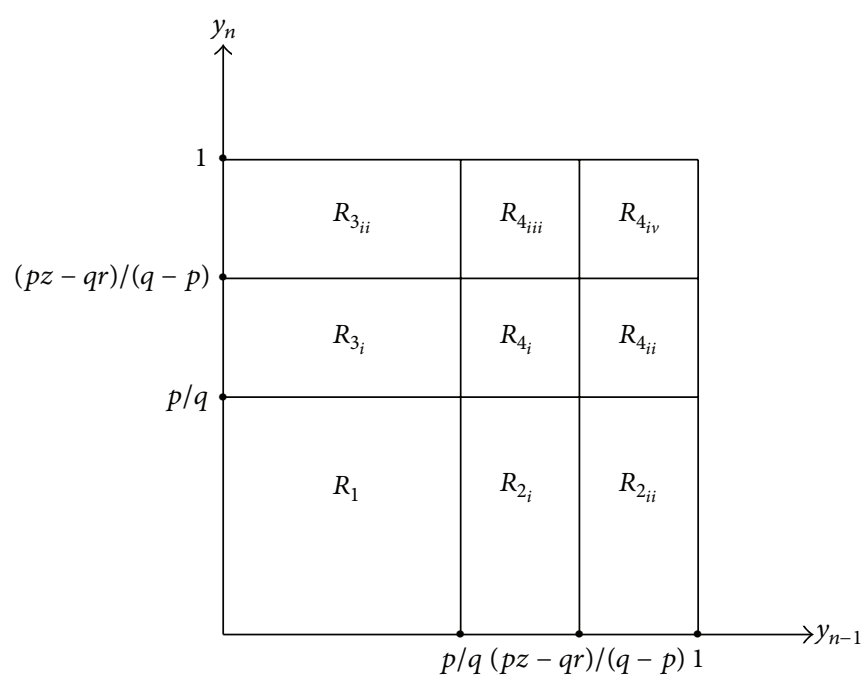

(b)

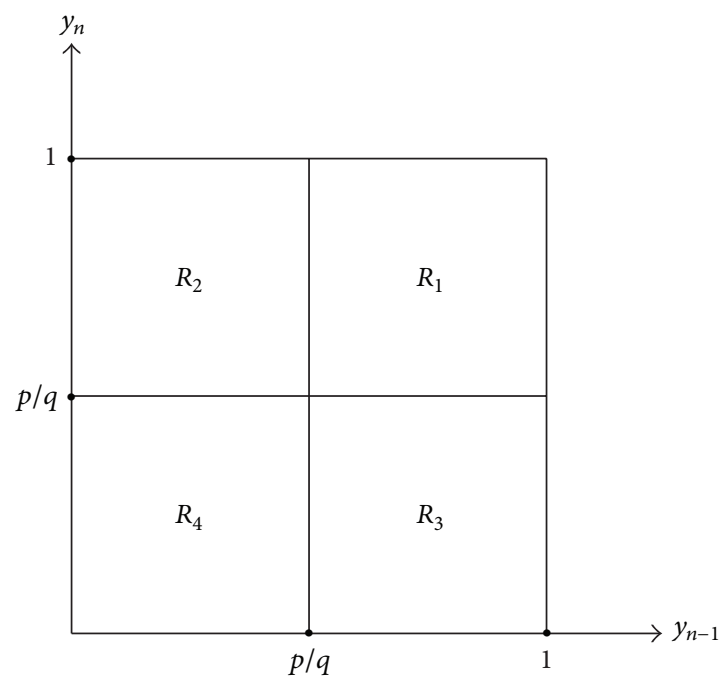

(c)

FIGURE 1: All possible regions in the plane $y_{n-1}-y_{n}$ when $p<q, r \leq z$, and $p z-q r>0$; (a) $(p z-q r) /(q-p)<p / q$; (b) $(p z-q r) /(q-p)>p / q$; (c) $(p z-q r) /(q-p)=p / q$.

(b) If $(p z-q r) /(q-p)>p / q$, then every positive solution of (6) eventually enters and remains in the interval $(0, p / q)$.

(c) If $(p z-q r) /(q-p)=p / q$, then every positive solution of (6) eventually enters and remains in the interval $[p / q, 1]$.

Proof. Assume that $p<q$ and $r \leq z$. Identity (51) shows that $y_{n}<1$ for all $n \geq 0$.

Here we have two cases to be considered.

(1) If $p z-q r \leq 0$. In view of Table 1 , Cases 2,3 , and 4 , the function $f\left(y_{n}, y_{n-1}\right)$ is increasing in $y_{n-1}$ and decreasing in $y_{n}$ for all values of $y_{n}$ and $y_{n-1}$. Using the decreasing character of $f$ in $y_{n}$, and the increasing character in $y_{n-1}$, we obtain

$$
\begin{aligned}
\frac{p}{q}<\frac{q(r+p)+p}{q(z+q)+p} & =f\left(1, \frac{p}{q}\right)<f\left(y_{n}, y_{n-1}\right)<f\left(\frac{p}{q}, 1\right) \\
& =\frac{q(r+1)+p^{2}}{q(z+1)+p q}<1 .
\end{aligned}
$$

(2) If $p z-q r>0$. In this case, the unit square $[0,1] \times[0,1]$ divides into the regions depicted in Figure 1.

We have to treat the cases: $(p z-q r) /(q-p)<p / q,(p z-$ $q r) /(q-p)>p / q$, and $(p z-q r) /(q-p)=p / q$. 


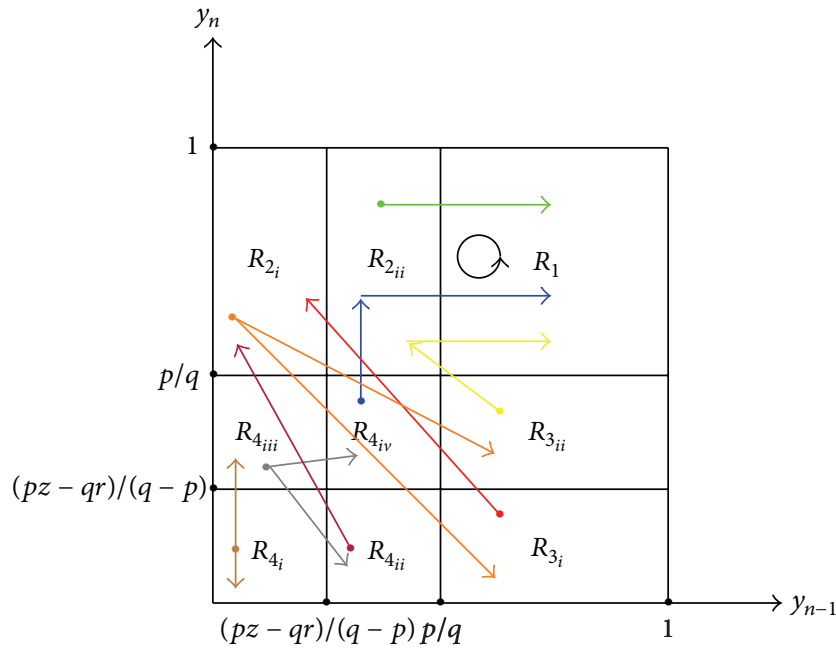

FIGURE 2: The dynamics of $\left(y_{n-1}, y_{n}\right)$ in the plane $y_{n-1}-y_{n}$ when $(p z-q r) /(q-p)<p / q$.

(a) $(p z-q r) /(q-p)<p / q$. In this case, the dynamics of $\left(y_{n-1}, y_{n}\right)$ are depicted in Figure 2 .

Assume that $\left(y_{n-1}, y_{n}\right) \in R_{1}$; then $p / q \leq y_{n-1}, y_{n} \leq 1$. Remark 11(2) implies $y_{n+1}>p / q$. Furthermore, by Table 1, Case 1 , the function $f\left(y_{n-1}, y_{n}\right)$ is increasing in $y_{n-1}$ and decreasing in $y_{n}$. Using the decreasing character of $f$ in $y_{n}$, and the increasing character in $y_{n-1}$, we obtain

$$
\begin{aligned}
\frac{p}{q}<\frac{q(r+p)+p}{q(z+q)+p} & =f\left(1, \frac{p}{q}\right)<f\left(y_{n}, y_{n-1}\right)<f\left(\frac{p}{q}, 1\right) \\
& =\frac{q(r+1)+p^{2}}{q(z+1)+p q}<1 .
\end{aligned}
$$

Which implies the invariance of the interval $[p / q, 1]$.

Now we will study the entrance to the interval.

By Remark 11(2) we have the following cases.

(1) $\left(y_{n-1}, y_{n}\right) \in R_{2_{i i}} \Rightarrow\left(y_{n}, y_{n+1}\right) \in R_{1}$.

(2) $\left(y_{n-1}, y_{n}\right) \in R_{3_{i i}} \cup R_{4_{i v}} \Rightarrow\left(y_{n}, y_{n+1}\right) \in R_{2_{i i}} \Rightarrow\left(y_{n+1}\right.$, $\left.y_{n+2}\right) \in R_{1}$.

(3) $\left(y_{n-1}, y_{n}\right) \in R_{4_{i i i}} \Rightarrow\left(y_{n}, y_{n+1}\right) \in R_{4_{i v}} \cup R_{4_{i i}}$.

(4) $\left(y_{n-1}, y_{n}\right) \in R_{4_{i}} \Rightarrow\left(y_{n}, y_{n+1}\right) \in R_{4_{i i i}} \cup R_{4_{i}}$.

(5) $\left(y_{n-1}, y_{n}\right) \in R_{4_{i i}} \cup R_{3_{i}} \Rightarrow\left(y_{n}, y_{n+1}\right) \in R_{2_{i}}$.

(6) $\left(y_{n-1}, y_{n}\right) \in R_{2_{i}} \Rightarrow\left(y_{n}, y_{n+1}\right) \in R_{3_{i i}} \cup R_{3_{i}}$.

The dynamics of $\left(y_{n-1}, y_{n}\right)$ using directed graph are depicted in Figure 3.

Our interest now is to show that the pairs $\left(y_{n-1}, y_{n}\right)$ cannot stay forever in $R_{4_{i}}$. Also, we need to show that the pairs $\left(y_{n-1}, y_{n}\right)$ cannot stay forever in $R_{2_{i}} \cup R_{3_{i}}$.

First assume that $\left(y_{n-1}, y_{n}\right) \in R_{4 i}$; then $y_{n-1}, y_{n}<(p z-$ $q r) /(q-p)$. In view of Table 1 , Case 1 , the solution increases in both arguments. Using the increasing character of $f$, we obtain

$$
\begin{aligned}
0<\frac{r}{z} & =f(0,0)<f\left(y_{n}, y_{n-1}\right)<f\left(\frac{p z-q r}{q-p}, \frac{p z-q r}{q-p}\right) \\
& =\frac{r(q-p)+(p+1)(p z-q r)}{z(q-p)+(q+1)(p z-q r)}=\frac{p}{q} .
\end{aligned}
$$

As such the limit of the solution lies in the interval $(0, p / q)$, which is impossible because $\bar{y} \geq p / q$ by Lemma 12 , part 1 . Hence every positive solution of (6) in the region $R_{4_{i}}$ also eventually enters and remains in the interval $[p / q, 1]$. $R_{3_{i}}$.

Next assume that $\left(y_{n-1}, y_{n}\right) \in R_{2_{i}}$; then $\left(y_{n}, y_{n+1}\right) \in R_{3_{i i}} \cup$ $R_{1}$.

If $\left(y_{n}, y_{n+1}\right) \in R_{3_{i i}} \Rightarrow\left(y_{n+1}, y_{n+2}\right) \in R_{2_{i i}} \Rightarrow\left(y_{n+2}, y_{n+3}\right) \in$

If $\left(y_{n}, y_{n+1}\right) \in R_{3_{i}} \Rightarrow\left(y_{n+1}, y_{n+2}\right) \in R_{2_{i}} \Rightarrow\left(y_{n+2}, y_{n+3}\right) \in$ $R_{3_{i i}} \cup R_{3_{i}}$ and so on.

Without loss of generality, we may assume that $y_{2 n+1}<$ $(p z-q r) /(q-p)$, whereas $y_{2 n}>p / q$ for all $n$. But,

$$
y_{2 n+1}=\frac{r+p y_{2 n}+y_{2 n-1}}{z+q y_{2 n}+y_{2 n-1}}>\frac{r+y_{2 n-1}}{z+y_{2 n-1}}>y_{2 n-1} .
$$

The first inequality holds true because $y_{2 n-1}<(p z-q r) /(q-$ $p)$. Furthermore, the second inequality follows from the facts that the graph of $g(x)=(r+x) /(z+x)$ looks like the one depicted in Figure 4, and

$$
g\left(\frac{p z-q r}{q-p}\right)=\frac{r(q-p)+(p z-q r)}{z(q-p)+(p z-q r)}=\frac{p}{q}>\frac{p z-q r}{q-p} .
$$

Thus the odd terms converge and so do the even terms. This implies the existence of a period-two solution which is a contradiction since, by Theorem 9, (6) does not possess a period-two solution.

(b) $(p z-q r) /(q-p)>p / q$. In this case, the dynamics of $\left(y_{n-1}, y_{n}\right)$ are depicted in Figure 5 .

Assume that $\left(y_{n-1}, y_{n}\right) \in R_{1}$; then $0<y_{n-1}, y_{n} \leq p / q<$ $(p z-q r) /(q-p)$. Remark 11(2) implies $y_{n+1}<p / q$. Furthermore, by Table 1 , Case 1 , the function $f\left(y_{n-1}, y_{n}\right)$ is increasing in $y_{n-1}$ and $y_{n}$. Using the increasing character of $f$, we obtain

$$
\begin{aligned}
0<\frac{r}{z} & =f(0,0)<f\left(y_{n}, y_{n-1}\right)<f\left(\frac{p z-q r}{q-p}, \frac{p z-q r}{q-p}\right) \\
& =\frac{r(q-p)+(p+1)(p z-q r)}{z(q-p)+(q+1)(p z-q r)}=\frac{p}{q},
\end{aligned}
$$

which implies the invariance of the interval $(0, p / q)$.

Now we will study the entrance to the interval.

By Remark 11(2) we have the following cases.

(1) $\left(y_{n-1}, y_{n}\right) \in R_{2_{i i}} \Rightarrow\left(y_{n}, y_{n+1}\right) \in R_{1}$.

(2) $\left(y_{n-1}, y_{n}\right) \in R_{3_{i i}} \cup R_{4_{i v}} \Rightarrow\left(y_{n}, y_{n+1}\right) \in R_{2_{i i}} \Rightarrow\left(y_{n+1}\right.$, $\left.y_{n+2}\right) \in R_{1}$. 


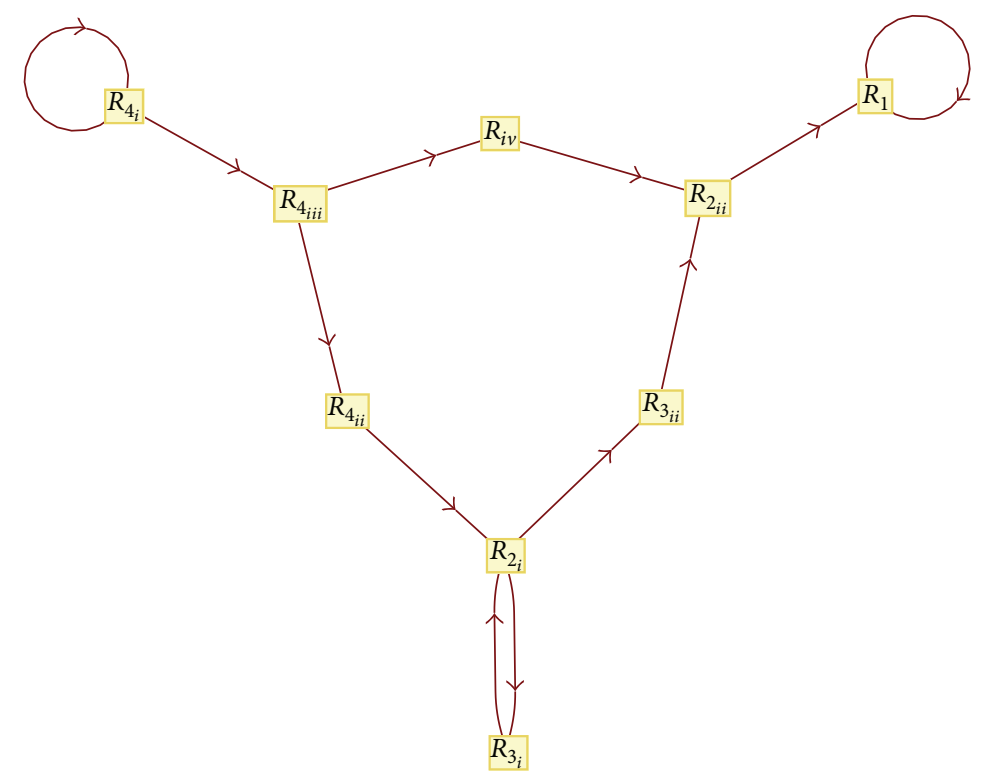

FIgURE 3: The dynamics of $\left(y_{n-1}, y_{n}\right)$ when $(p z-q r) /(q-p)<p / q$.

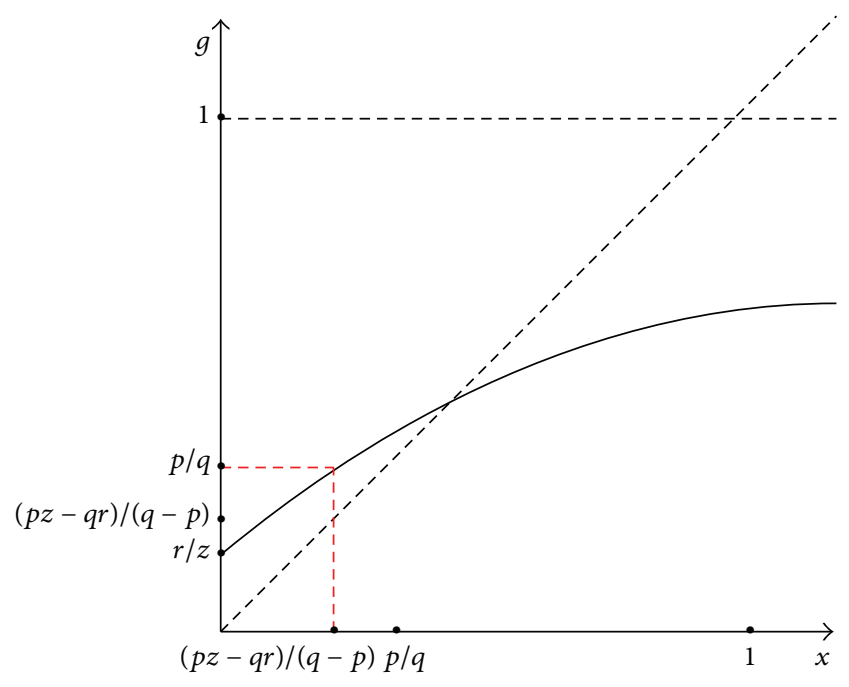

FIGURE 4: $g(x)=(r+x) /(z+x)$ when $r \leq z$ and $(p z-q r) /(q-p)<$ $p / q$.

(3) $\left(y_{n-1}, y_{n}\right) \in R_{4_{i i i}} \Rightarrow\left(y_{n}, y_{n+1}\right) \in R_{4_{i v}} \cup R_{4_{i i}}$.

(4) $\left(y_{n-1}, y_{n}\right) \in R_{4_{i}} \Rightarrow\left(y_{n}, y_{n+1}\right) \in R_{4_{i i i}} \cup R_{4_{i}}$.

(5) $\left(y_{n-1}, y_{n}\right) \in R_{4_{i i}} \cup R_{3_{i}} \Rightarrow\left(y_{n}, y_{n+1}\right) \in R_{2_{i}}$.

(6) $\left(y_{n-1}, y_{n}\right) \in R_{2_{i}} \Rightarrow\left(y_{n}, y_{n+1}\right) \in R_{3_{i i}} \cup R_{3_{i}}$.

Our interest now is to show that the pairs $\left(y_{n-1}, y_{n}\right)$ cannot stay forever in $R_{4_{i}}$. Also, we need to show that the pairs $\left(y_{n-1}, y_{n}\right)$ cannot stay forever in $R_{2_{i}} \cup R_{3_{i}}$.

First assume that $\left(y_{n-1}, y_{n}\right) \in R_{4_{i}}$; then $y_{n-1}, y_{n}>$ $(p z-q r) /(q-p)$. In view of Table 1 , Case 1 , the function $f\left(y_{n}, y_{n-1}\right)$ is increasing in $y_{n-1}$ and decreasing in $y_{n}$. Using

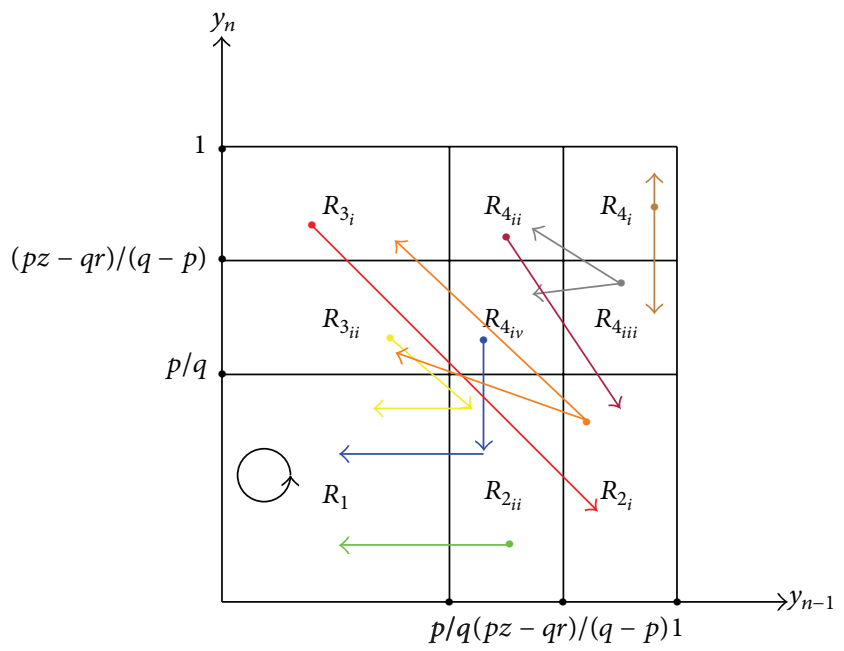

FIgURE 5: The dynamics of $\left(y_{n-1}, y_{n}\right)$ in the plane $y_{n-1}-y_{n}$ when $(p z-q r) /(q-p)>p / q$.

the decreasing character of $f$ in $y_{n}$, and the increasing character in $y_{n-1}$, we obtain

$$
\begin{aligned}
\frac{p z-q r}{q-p} & <\frac{(r+p)(q-p)+p z-q r}{(z+q)(q-p)+p z-q r} \\
& =f\left(1, \frac{p z-q r}{q-p}\right)<f\left(y_{n}, y_{n-1}\right)<f\left(\frac{p z-q r}{q-p}, 1\right) \\
& =\frac{(r+1)(q-p)+p(p z-q r)}{(z+1)(q-p)+q(p z-q r)}<1 .
\end{aligned}
$$




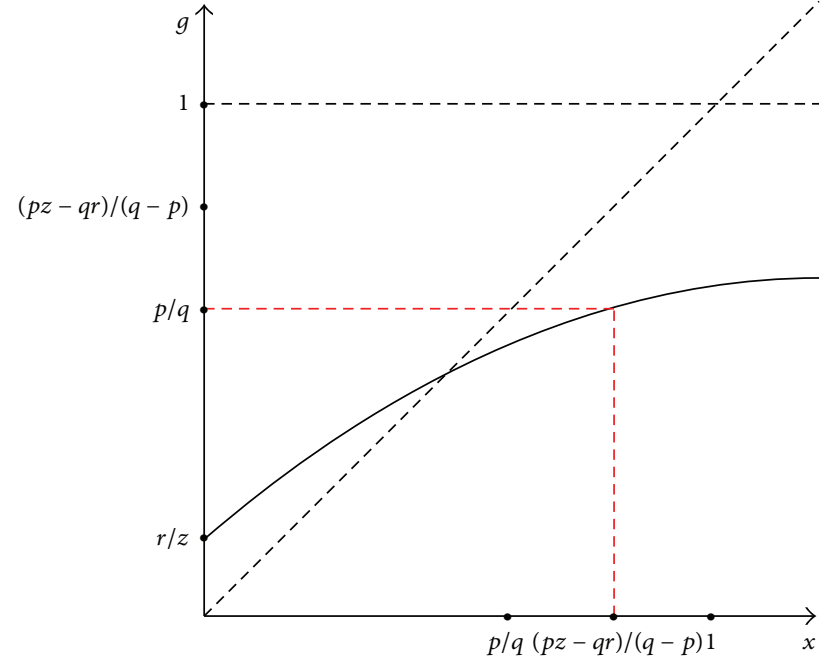

Figure 6: $g(x)=(r+x) /(z+x)$ when $r \leq z$ and $(p z-q r) /(q-p)>$ $p / q$.

As such the limit of the solution lies in the interval $[(p z-q r) /(q-p), 1]$, which is impossible because $\bar{y}<p / q$ by Lemma 12 , part 2 . Hence every positive solution of (6) in the region $R_{4}$ also eventually enters and remains in the interval $(0, p / q)$.

Next assume that $\left(y_{n-1}, y_{n}\right) \in R_{2_{i}}$; then $\left(y_{n}, y_{n+1}\right) \in$ $R_{3_{i i}} \cup R_{3_{i}}$. $R_{1}$.

If $\left(y_{n}, y_{n+1}\right) \in R_{3_{i i}} \Rightarrow\left(y_{n+1}, y_{n+2}\right) \in R_{2_{i i}} \Rightarrow\left(y_{n+2}, y_{n+3}\right) \in$

If $\left(y_{n}, y_{n+1}\right) \in R_{3_{i}} \Rightarrow\left(y_{n+1}, y_{n+2}\right) \in R_{2_{i}} \Rightarrow\left(y_{n+2}, y_{n+3}\right) \in$ $R_{3_{i i}} \cup R_{3_{i}}$ and so on.

Without loss of generality, we may assume that $y_{2 n+1}>(p z-q r) /(q-p)$, whereas $y_{2 n}<p / q$ for all $n$. But,

$$
y_{2 n+1}=\frac{r+p y_{2 n}+y_{2 n-1}}{z+q y_{2 n}+y_{2 n-1}}<\frac{r+y_{2 n-1}}{z+y_{2 n-1}}<y_{2 n-1} .
$$

The first inequality holds true because $y_{2 n-1}>(p z-q r) /(q-$ $p)$. Furthermore, the second inequality follows from the facts that the graph of $g(x)=(r+x) /(z+x)$ looks like the one depicted in Figure 6, and

$$
g\left(\frac{p z-q r}{q-p}\right)=\frac{r(q-p)+(p z-q r)}{z(q-p)+(p z-q r)}=\frac{p}{q}<\frac{p z-q r}{q-p} .
$$

Thus the odd terms converge and so do the even terms. This implies the existence of a period-two solution which is a contradiction since, by Theorem 9, (6) does not possess a period-two solution.

(c) $(p z-q r) /(q-p)=p / q$. In this case, the dynamics of $\left(y_{n-1}, y_{n}\right)$ are depicted in Figure 7 .

Assume that $\left(y_{n-1}, y_{n}\right) \in R_{1}$; then $p / q \leq y_{n-1}, y_{n} \leq 1$. Remark 11(2) implies $y_{n+1}>p / q$. Furthermore, by Table 1, Case 1 , the function $f\left(y_{n-1}, y_{n}\right)$ is increasing in $y_{n-1}$ and

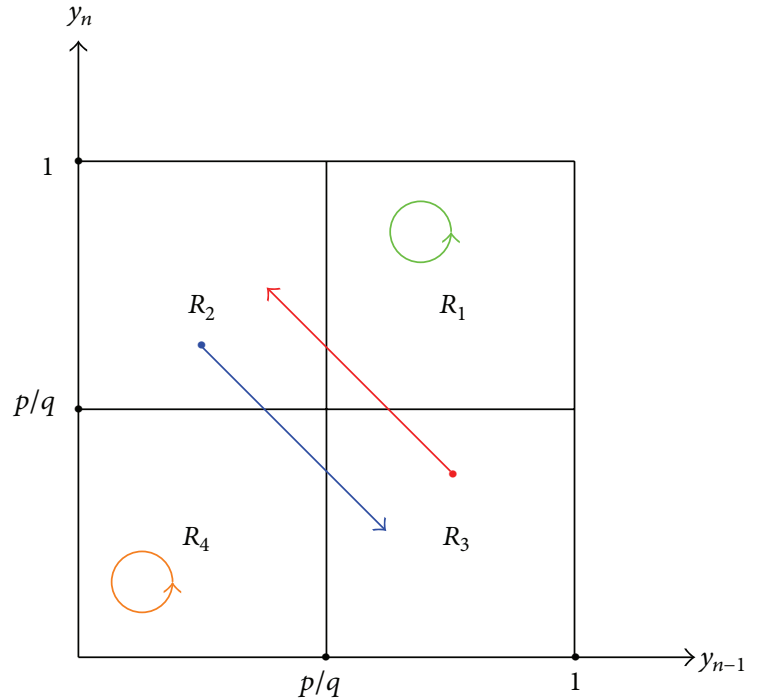

Figure 7: The dynamics of $\left(y_{n-1}, y_{n}\right)$ in the plane $y_{n-1}-y_{n}$ when $(p z-q r) /(q-p)=p / q$.

decreasing in $y_{n}$. Using the decreasing character of $f$ in $y_{n}$, and the increasing character in $y_{n-1}$, we obtain

$$
\begin{aligned}
\frac{p}{q}<\frac{q(r+p)+p}{q(z+q)+p} & =f\left(1, \frac{p}{q}\right)<f\left(y_{n}, y_{n-1}\right)<f\left(\frac{p}{q}, 1\right) \\
& =\frac{q(r+1)+p^{2}}{q(z+1)+p q}<1,
\end{aligned}
$$

which implies the invariance of the interval $[p / q, 1]$.

By Remark 11(2) we have the following cases.

(1) $\left(y_{n-1}, y_{n}\right) \in R_{2} \Rightarrow\left(y_{n}, y_{n+1}\right) \in R_{3}$.

(2) $\left(y_{n-1}, y_{n}\right) \in R_{3} \Rightarrow\left(y_{n}, y_{n+1}\right) \in R_{2}$.

(3) $\left(y_{n-1}, y_{n}\right) \in R_{4} \Rightarrow\left(y_{n}, y_{n+1}\right) \in R_{4}$.

Our interest now is to show that the pairs $\left(y_{n-1}, y_{n}\right)$ cannot stay forever in $R_{4}$. Also, we need to show that the pairs $\left(y_{n-1}, y_{n}\right)$ cannot stay forever in $R_{2} \cup R_{3}$.

First assume that $\left(y_{n-1}, y_{n}\right) \in R_{4}$; then $y_{n-1}, y_{n}<p / q=$ $(p z-q r) /(q-p)$. In view of Table 1 , Case 1 , the solution increases in both arguments. Using the increasing character of $f$, we obtain

$$
\begin{aligned}
0<\frac{r}{z} & =f(0,0)<f\left(y_{n}, y_{n-1}\right)<f\left(\frac{p z-q r}{q-p}, \frac{p z-q r}{q-p}\right) \\
& =\frac{r(q-p)+(p+1)(p z-q r)}{z(q-p)+(q+1)(p z-q r)}=\frac{p}{q} .
\end{aligned}
$$

As such the limit of the solution lies in the interval $(0, p / q)$, which is impossible because $\bar{y}=p / q$ by Lemma 12 . Hence every positive solution of (6) in the region $R_{4}$ also eventually enters and remains in the interval $[p / q, 1]$. 
Next assume that $\left(y_{n-1}, y_{n}\right) \in R_{2}$; then $\left(y_{n}, y_{n+1}\right) \in R_{3}$ and vice versa.

Without loss of generality, we may assume that $y_{2 n+1}<$ $(p z-q r) /(q-p)$, whereas $y_{2 n} \geq(p z-q r) /(q-p)$ for all $n$. But,

$$
y_{2 n+1}=\frac{r+p y_{2 n}+y_{2 n-1}}{z+q y_{2 n}+y_{2 n-1}}>\frac{r+y_{2 n-1}}{z+y_{2 n-1}}=y_{2 n-1} .
$$

The first inequality holds true because $y_{2 n-1}<(p z-q r) /$ $(q-p)$. Furthermore, the second equality follows from the facts that $g(x)=(r+x) /(z+x)$ at $(p z-q r) /(q-p)$ is

$$
g\left(\frac{p z-q r}{q-p}\right)=\frac{r(q-p)+(p z-q r)}{z(q-p)+(p z-q r)}=\frac{p}{q}=\frac{p z-q r}{q-p}
$$

Thus the odd terms converge and so do the even terms. This implies the existence of a period-two solution which is a contradiction since, by Theorem 9, (6) does not possess a period-two solution.

The proof is complete.

Lemma 14. Assume that $p<q$ and $r>z$.

(1) If $(r-z) /(q-p) \leq 1$ then $\bar{y} \leq 1$.

(2) If $(r-z) /(q-p)>1$ then $\bar{y}>1$.

Proof. We will prove (1); the proof of (2) is similar and will be omitted. By (34), making use of $(r-z) /(q-p) \leq 1 \Rightarrow r \leq$ $-p+z, \bar{y}$ is such that

$$
\bar{y} \leq \frac{1+p-z+\sqrt{(1+p-z)^{2}+4(q-p+z)(q+1)}}{2(q+1)} .
$$

After some elementary algebraic manipulation, (68) is equivalent to

$$
\begin{aligned}
\bar{y} & \leq \frac{1+p-z+\sqrt{(1-p+z)^{2}+4 q^{2}+4 q(1-p+z)}}{2(q+1)} \\
& \leq \frac{1+p-z+\sqrt{(1-p+z+2 q)^{2}}}{2(q+1)} \\
& \leq \frac{1+p-z+(1-p+z+2 q)}{2(q+1)}=1 .
\end{aligned}
$$

The proof is complete.

By Remark 11, Lemma 14, and Table 1, we obtain the following key result.

Theorem 15. Assume that $p<q$ and $r>z$; then we have two cases to be considered.

(1) If $(r-z) /(q-p)<1$, then every positive solution of (6) eventually enters and remains in the interval $[(r-$ $z) /(q-p), 1]$.
(2) If $(r-z) /(q-p)>1$, then every positive solution of (6) eventually enters and remains in the interval $[1,(r-$ $z) /(q-p)]$.

(3) If $(r-z) /(q-p)=1$, then every positive solution of (6) eventually enters and remains in the interval $[(r-$ $z) /(q-p), 1]$.

Proof. Assume that $p<q$ and $r>z$. Identity (52) shows that $y_{n}>p / q$ for all $n \geq 0$. In this case, the plane $y_{n-1}-y_{n}$ divides into the regions depicted in Figure 8.

We have to treat the cases $(r-z) /(q-p)<1,(r-z) /(q-$ $p)>1$, and $(r-z) /(q-p)=1$.

(1) $(r-z) /(q-p)<1$. In this case, the dynamics of $\left(y_{n-1}, y_{n}\right)$ are depicted in Figure 9.

Assume that $\left(y_{n-1}, y_{n}\right) \in R_{4_{i v}}$; then $(r-z) /(q-p) \leq y_{n-1}$, $y_{n} \leq 1$. In this case, by Table 1 , Case 5 , the function $f\left(y_{n-1}\right.$, $y_{n}$ ) is increasing in $y_{n-1}$ and decreasing in $y_{n}$. Using the decreasing character of $f$ in $y_{n}$, and the increasing character in $y_{n-1}$, we obtain

$$
\begin{aligned}
\frac{r-z}{q-p} & <\frac{(r+p)(q-p)+(r-z)}{(z+q)(q-p)+(r-z)} \\
& =f\left(1, \frac{r-z}{q-p}\right)<f\left(y_{n}, y_{n-1}\right)<f\left(\frac{r-z}{q-p}, 1\right) \\
& =\frac{(r+1)(q-p)+p(r-z)}{(z+1)(q-p)+q(r-z)}=1,
\end{aligned}
$$

which implies the invariance of the interval $[(r-z) /(q-p), 1]$.

Now we will study the entrance to the interval.

By Remark 11(2) we have the following cases.

$$
\begin{aligned}
& \text { (1) }\left(y_{n-1}, y_{n}\right) \in R_{4_{i i i}} \cup R_{3_{i i}} \Rightarrow\left(y_{n}, y_{n+1}\right) \in R_{4_{i v}} \cup R_{4_{i i}} . \\
& \text { (2) }\left(y_{n-1}, y_{n}\right) \in R_{1} \cup R_{2_{i}} \cup R_{2_{i i}} \Rightarrow\left(y_{n}, y_{n+1}\right) \in R_{3_{i i}} \cup R_{3_{i}} . \\
& \text { (3) }\left(y_{n-1}, y_{n}\right) \in R_{4_{i}} \cup R_{4_{i i}} \cup R_{3_{i}} \Rightarrow\left(y_{n}, y_{n+1}\right) \in R_{2_{i}} .
\end{aligned}
$$

The dynamics of $\left(y_{n-1}, y_{n}\right)$ using directed graph are depicted in Figure 10.

From the directed graph, we can see that if a solution is not eventually in $[(r-z) /(q-p), 1]$, it converges to a periodic solution with period 2 or 3 .

Our interest now is to show that the pairs $\left(y_{n-1}, y_{n}\right)$ cannot stay forever in $R_{2_{i}} \cup R_{3_{i}}$. Also, we need to show that the pairs $\left(y_{n-1}, y_{n}\right)$ cannot stay forever in $R_{2_{i}} \cup R_{3_{i i}} \cup R_{4_{i i}}$.

First, assume that $y_{2 n}<(r-z) /(q-p)$, whereas $y_{2 n-1}>1$ for all $n$. Then

$$
\begin{aligned}
y_{2 n+1}-y_{2 n-1} & <\frac{r+p y_{2 n}}{z+q y_{2 n}}-y_{2 n-1} \\
& <\frac{\left(r-z y_{2 n-1}\right)+\left(p-q y_{2 n-1}\right) y_{2 n}}{z+q y_{2 n}} \\
& <\frac{q y_{2 n-1}-p}{z+q y_{2 n}}\left[\frac{r-z y_{2 n-1}}{q y_{2 n-1}-p}-y_{2 n}\right] .
\end{aligned}
$$




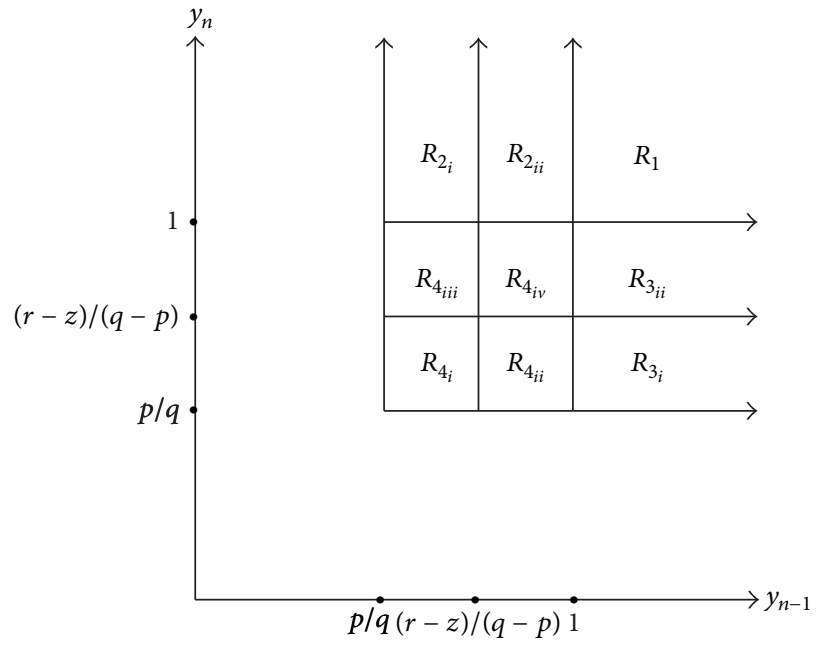

(a)

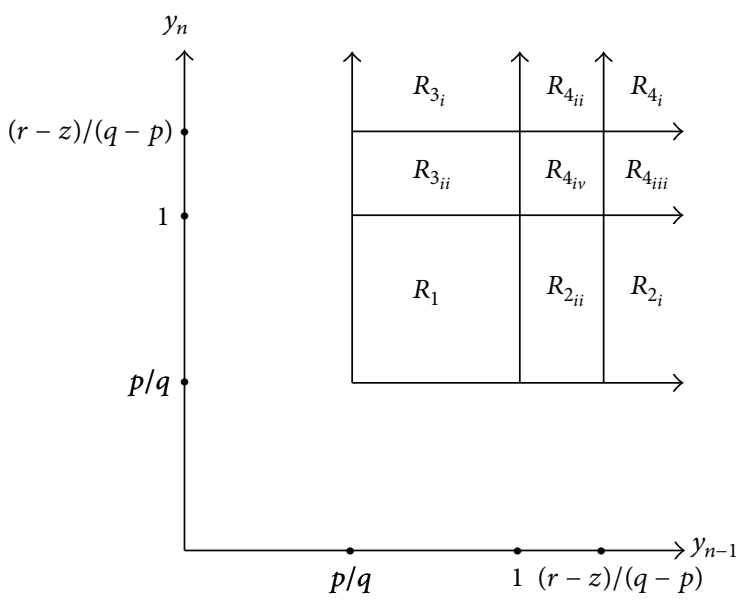

(b)

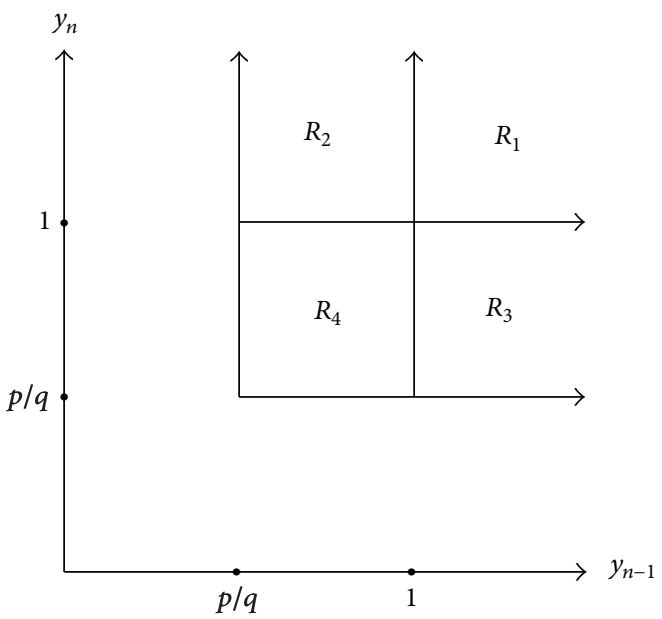

(c)

FIGURE 8: All possible regions in the plane $y_{n-1}-y_{n}$ when $p<q$ and $r>z ;(\mathrm{a})(r-z) /(q-p)<1 ;(\mathrm{b})(r-z) /(q-p)>1 ;(\mathrm{c})(r-z) /(q-p)=1$.

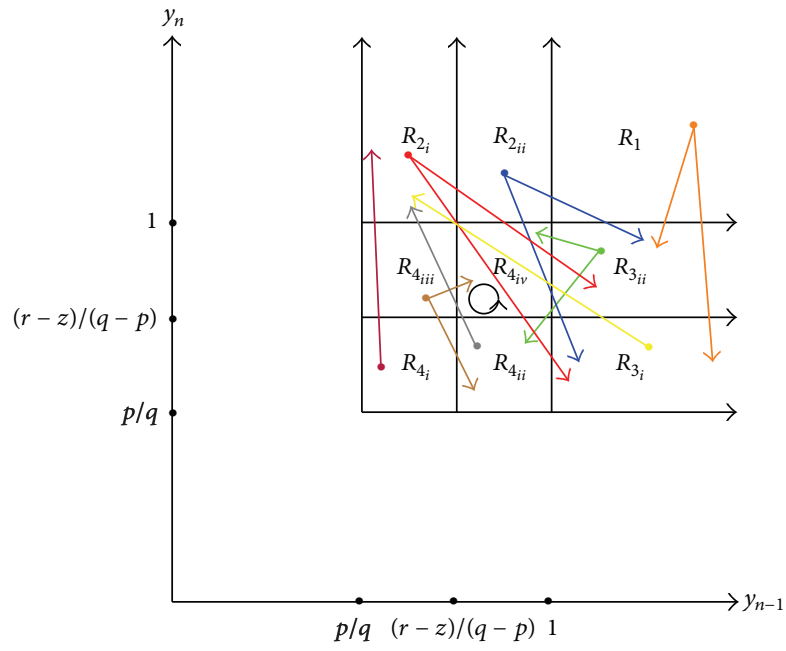

FIGURE 9: The dynamics of $\left(y_{n-1}, y_{n}\right)$ in the plane $y_{n-1}-y_{n}$ when $(r-z) /(q-p)<1$.
Since $p<q, r>z$, and $y_{2 n-1}>1$, then $\left(r-z y_{2 n-1}\right) /\left(q y_{2 n-1}-\right.$ $p)<(r-z) /(q-p)$. Furthermore, $y_{2 n}<(r-z) /(q-p)$, so the solution is decreasing. With that in mind,

$$
\begin{aligned}
y_{2 n+1}-y_{2 n-1}< & \frac{q y_{2 n-1}-p}{z+q y_{2 n}}\left[\frac{r-z}{q-p}-y_{2 n}\right] \\
< & \frac{q(1)-p}{z+q((r-z) /(q-p))} \\
& \cdot\left[\frac{r-z}{q-p}-\frac{r-z}{q-p}\right]=0 .
\end{aligned}
$$

Thus the odd terms converge and so do the even terms. This implies the existence of a period-two solution which is a contradiction since, by Theorem 9, (6) does not possess a period-two solution. 


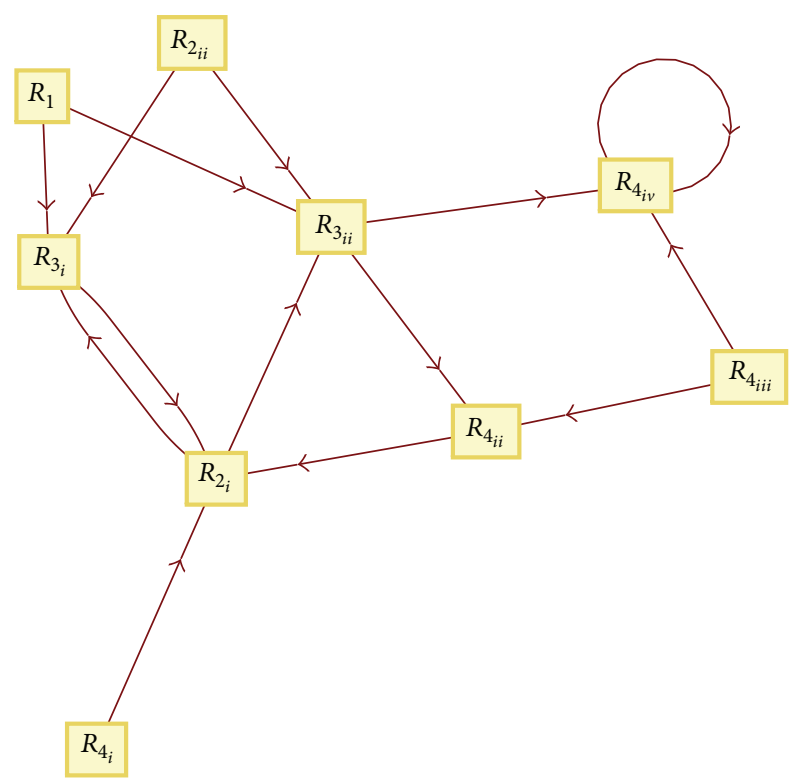

Figure 10: The dynamics of $\left(y_{n-1}, y_{n}\right)$ when $(r-z) /(q-p)<1$.

Next, assume that $y_{3 n}<(r-z) /(q-p), y_{3 n-2}>1$, whereas $(r-z) /(q-p)<y_{3 n-1}<1$ for all $n$. Then

$$
\begin{aligned}
y_{3 n+1}-y_{3 n-2} & <\frac{r+p y_{3 n}}{z+q y_{3 n}}-y_{3 n-2} \\
& <\frac{\left(r-z y_{3 n-2}\right)+\left(p-q y_{3 n-2}\right) y_{3 n}}{z+q y_{3 n}} \\
& <\frac{q y_{3 n-2}-p}{z+q y_{3 n}}\left[\frac{r-z y_{3 n-2}}{q y_{3 n-2}-p}-y_{3 n}\right] .
\end{aligned}
$$

Since $p<q, r>z$, and $y_{3 n-2}>1$ then $\left(r-z y_{3 n-1}\right) /\left(q y_{3 n-1}-\right.$ $p)<(r-z) /(q-p)$. Furthermore, $y_{3 n}<(r-z) /(q-p)$, so the solution is decreasing. With that in mind,

$$
\begin{aligned}
y_{3 n+1}-y_{3 n-2}< & \frac{q y_{3 n-2}-p}{z+q y_{3 n}}\left[\frac{r-z}{q-p}-y_{3 n}\right] \\
< & \frac{q(1)-p}{z+q((r-z) /(q-p))} \\
& \cdot\left[\frac{r-z}{q-p}-\frac{r-z}{q-p}\right]=0 .
\end{aligned}
$$

Thus the subsequences $\left\{y_{3 n-2}\right\},\left\{y_{3 n-1}\right\}$, and $\left\{y_{3 n}\right\}$ converge to finite limits say, $L_{1}, L_{2}$, and $L_{3}$. Set

$$
\begin{aligned}
& L_{1}=\lim _{n \rightarrow \infty} y_{3 n-1}, \\
& L_{2}=\lim _{n \rightarrow \infty} y_{3 n-2}, \\
& L_{3}=\lim _{n \rightarrow \infty} y_{3 n-3} .
\end{aligned}
$$

Then

$$
\ldots, L_{1}, L_{2}, L_{3} \ldots
$$

is a periodic solution of (6) with period-three. By (6),

$$
\begin{aligned}
& L_{1}=\frac{r+p L_{3}+L_{2}}{z+q L_{3}+L_{2}}, \\
& L_{2}=\frac{r+p L_{1}+L_{3}}{z+q L_{1}+L_{3}}, \\
& L_{3}=\frac{r+p L_{2}+L_{1}}{z+q L_{2}+L_{1}} .
\end{aligned}
$$

Furthermore,

$$
\begin{aligned}
& L_{1}\left(z+q L_{3}+L_{2}\right)=r+p L_{3}+L_{2}, \\
& L_{2}\left(z+q L_{1}+L_{3}\right)=r+p L_{1}+L_{3}, \\
& L_{3}\left(z+q L_{2}+L_{1}\right)=r+p L_{2}+L_{1} .
\end{aligned}
$$

First, subtracting (80) from (78), we have

$$
z\left(L_{3}-L_{1}\right)+\left(q L_{3}+1\right)\left(L_{2}-L_{1}\right)+\left(L_{1}+p\right)\left(L_{3}-L_{2}\right)=0 .
$$

Next, subtracting (80) from (79), we have

$$
z\left(L_{3}-L_{2}\right)+\left(q L_{2}+1\right)\left(L_{3}-L_{1}\right)+\left(L_{3}+p\right)\left(L_{1}-L_{2}\right)=0 .
$$

Finally, subtracting (81) from (82), we have

$$
z\left(L_{2}-L_{1}\right)+\left(q L_{1}+1\right)\left(L_{2}-L_{3}\right)+\left(L_{2}+p\right)\left(L_{3}-L_{1}\right)=0 .
$$

But, under the assumption $y_{n-3}<(r-z) /(q-p), y_{n-2}>1$, whereas $(r-z) /(q-p)<y_{n-1}<1$, clearly

$$
L_{3}<L_{1}<L_{2} \text {. }
$$

As such, the left-hand side of (83) is positive, which is a contradiction.

(2) $(r-z) /(q-p)>1$. In this case, the dynamics of $\left(y_{n-1}, y_{n}\right)$ are depicted in Figure 11.

Assume that $\left(y_{n-1}, y_{n}\right) \in R_{4}$; then $1 \leq y_{n-1}, y_{n} \leq$ $(r-z) /(q-p)$. In this case, by Table 1 , Case 5 , the function $f\left(y_{n-1}, y_{n}\right)$ is decreasing in $y_{n-1}$ and decreasing in $y_{n}$. Using the decreasing character of $f$ in $y_{n}$ and $y_{n-1}$, we obtain

$$
\begin{aligned}
1 & =\frac{r(q-p)+(r-z)(p+1)}{z(q-p)+(r-z)(q+1)} \\
& =f\left(\frac{r-z}{q-p}, \frac{r-z}{q-p}\right)<f\left(y_{n}, y_{n-1}\right)<f(1,1) \\
& =\frac{r+p+1}{z+q+1}=\frac{r-z+z+p+1}{q-p+z+p+1}<\frac{r-z}{q-p},
\end{aligned}
$$

which implies the invariance of the interval $[1,(r-z) /(q-p)]$.

Now we will study the entrance to the interval.

By Remark 11(2) we have the following cases.

(1) $\left(y_{n-1}, y_{n}\right) \in R_{4_{i i i}} \cup R_{3_{i i}} \Rightarrow\left(y_{n}, y_{n+1}\right) \in R_{4_{i v}} \cup R_{4_{i i}}$. 


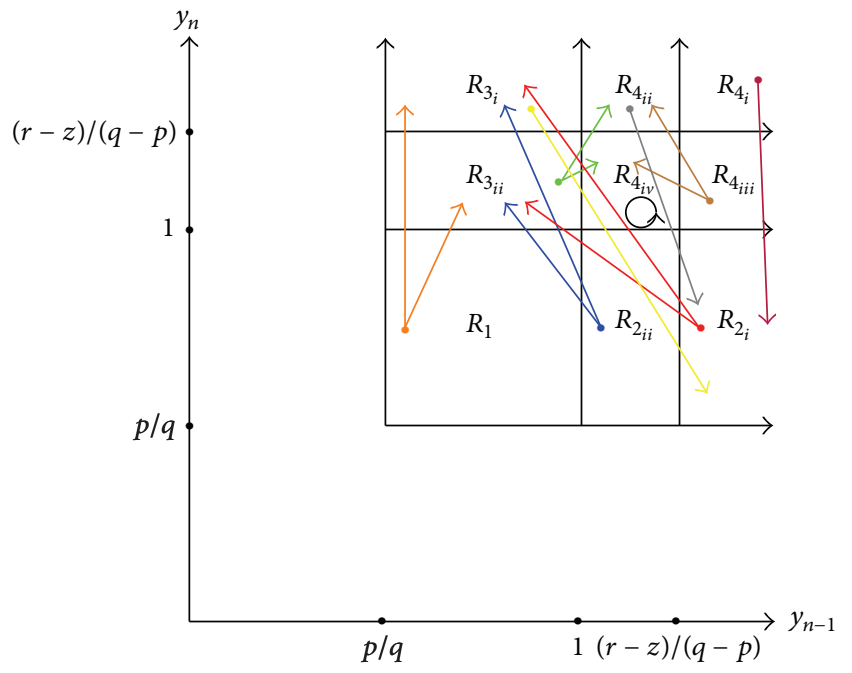

FIGURE 11: The dynamics of $\left(y_{n-1}, y_{n}\right)$ in the plane $y_{n-1}-y_{n}$ when $(r-z) /(q-p)>1$.

(2) $\left(y_{n-1}, y_{n}\right) \in R_{1} \cup R_{2_{i}} \cup R_{2_{i i}} \Rightarrow\left(y_{n}, y_{n+1}\right) \in R_{3_{i i}} \cup R_{3_{i}}$.

(3) $\left(y_{n-1}, y_{n}\right) \in R_{4_{i}} \cup R_{4_{i i}} \cup R_{3_{i}} \Rightarrow\left(y_{n}, y_{n+1}\right) \in R_{2_{i}}$.

The dynamics of $\left(y_{n-1}, y_{n}\right)$ using directed graph are depicted in Figure 10.

From the directed graph, we can see that if a solution is not eventually in $[1,(r-z) /(q-p)]$, it converges to a periodic solution with period 2 or 3 .

Our interest now is to show that the pairs $\left(y_{n-1}, y_{n}\right)$ cannot stay forever in $R_{2,} \cup R_{3_{i}}$. Also, we need to show that the pairs $\left(y_{n-1}, y_{n}\right)$ cannot stay forever in $R_{2_{i}} \cup R_{3_{i i}} \cup R_{4_{i i}}$.

First, assume that $y_{2 n}>(r-z) /(q-p)$, whereas $y_{2 n-1}<1$ for all $n$. Then

$$
\begin{aligned}
y_{2 n+1}-y_{2 n-1} & >\frac{r+p y_{2 n}}{z+q y_{2 n}}-y_{2 n-1} \\
& >\frac{\left(r-z y_{2 n-1}\right)+\left(p-q y_{2 n-1}\right) y_{2 n}}{z+q y_{2 n}} \\
& >\frac{q y_{2 n-1}-p}{z+q y_{2 n}}\left[\frac{r-z y_{2 n-1}}{q y_{2 n-1}-p}-y_{2 n}\right] .
\end{aligned}
$$

Since $p<q, r>z$, and $y_{2 n-1}<1$ then $\left(r-z y_{2 n-1}\right) /$ $\left(q y_{2 n-1}-p\right)>(r-z) /(q-p)$. Furthermore, $y_{2 n}>(r-z) /(q-p)$, so the solution is decreasing in $y_{n}$ and increasing in $y_{n-1}$. With that in mind,

$$
\begin{aligned}
y_{2 n+1}-y_{2 n-1}> & \frac{q y_{2 n-1}-p}{z+q y_{2 n}}\left[\frac{r-z}{q-p}-y_{2 n}\right] \\
> & \frac{q(1)-p}{z+q((r-z) /(q-p))} \\
& \cdot\left[\frac{r-z}{q-p}-\frac{r-z}{q-p}\right]=0 .
\end{aligned}
$$

Thus the odd terms converge and so do the even terms. This implies the existence of a period-two solution which is a contradiction since, by Theorem 9, (6) does not possess a period-two solution.

Next, assume that $y_{3 n}>(r-z) /(q-p), y_{3 n-2}<1$, whereas $1<y_{3 n-1}<(r-z) /(q-p)$ for all $n$. Then

$$
\begin{aligned}
y_{3 n+1}-y_{3 n-2} & >\frac{r+p y_{3 n}}{z+q y_{3 n}}-y_{3 n-2} \\
& >\frac{\left(r-z y_{3 n-2}\right)+\left(p-q y_{3 n-2}\right) y_{3 n}}{z+q y_{3 n}} \\
& >\frac{q y_{3 n-2}-p}{z+q y_{3 n}}\left[\frac{r-z y_{3 n-2}}{q y_{3 n-2}-p}-y_{3 n}\right] .
\end{aligned}
$$

Since $p<q, r>z$, and $y_{3 n-2}<1$ then $\left(r-z y_{3 n-1}\right) /\left(q y_{3 n-1}-\right.$ $p)>(r-z) /(q-p)$. Furthermore, $y_{3 n}>(r-z) /(q-p)$, so the solution is decreasing in $y_{n}$ and increasing in $y_{n-1}$. With that in mind,

$$
\begin{aligned}
y_{3 n+1}-y_{3 n-2}> & \frac{q y_{3 n-2}-p}{z+q y_{3 n}}\left[\frac{r-z}{q-p}-y_{3 n}\right] \\
> & \frac{q(1)-p}{z+q((r-z) /(q-p))} \\
& \cdot\left[\frac{r-z}{q-p}-\frac{r-z}{q-p}\right]=0 .
\end{aligned}
$$

Thus the subsequences $\left\{y_{3 n-2}\right\},\left\{y_{3 n-1}\right\}$, and $\left\{y_{3 n}\right\}$ converge to finite limits say, $J_{1}, J_{2}$, and $J_{3}$. Set

$$
\begin{aligned}
& J_{1}=\lim _{n \rightarrow \infty} y_{3 n-1}, \\
& J_{2}=\lim _{n \rightarrow \infty} y_{3 n-2}, \\
& J_{3}=\lim _{n \rightarrow \infty} y_{3 n-3} .
\end{aligned}
$$

Then

$$
\ldots, J_{1}, J_{2}, J_{3} \ldots
$$

is a periodic solution of (6) with period-three. By (6),

$$
\begin{aligned}
& J_{1}=\frac{r+p J_{3}+J_{2}}{z+q J_{3}+J_{2}}, \\
& J_{2}=\frac{r+p J_{1}+J_{3}}{z+q J_{1}+J_{3}}, \\
& J_{3}=\frac{r+p J_{2}+J_{1}}{z+q J_{2}+J_{1}} .
\end{aligned}
$$

Furthermore,

$$
\begin{aligned}
& J_{1}\left(z+q J_{3}+J_{2}\right)=r+p J_{3}+J_{2}, \\
& J_{2}\left(z+q J_{1}+J_{3}\right)=r+p J_{1}+J_{3}, \\
& J_{3}\left(z+q J_{2}+J_{1}\right)=r+p J_{2}+J_{1} .
\end{aligned}
$$

First, subtracting (95) from (93), we have

$$
z\left(J_{3}-J_{1}\right)+\left(q J_{3}+1\right)\left(J_{2}-J_{1}\right)+\left(J_{1}+p\right)\left(J_{3}-J_{2}\right)=0 .
$$




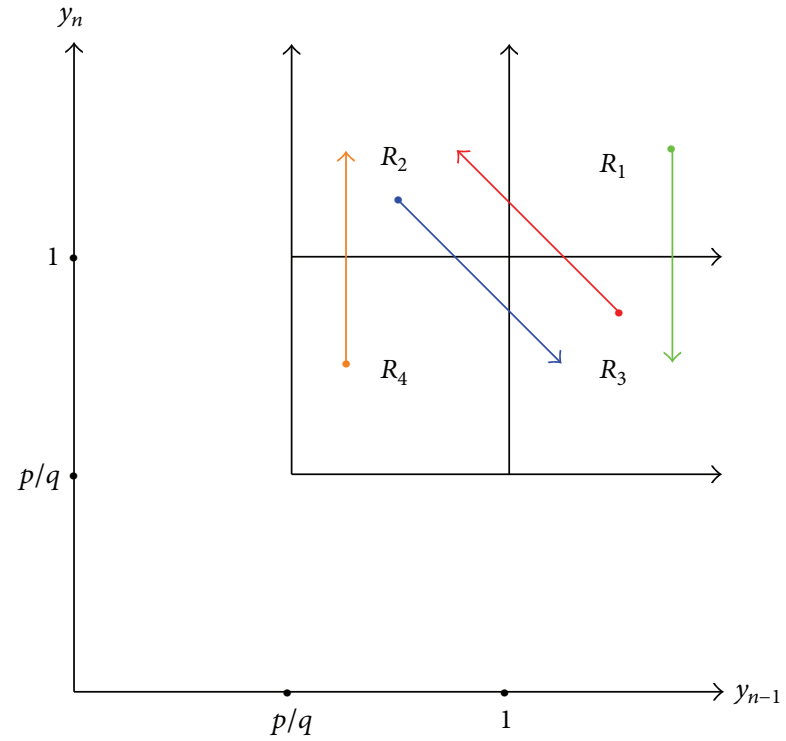

FIGURE 12: The dynamics of $\left(y_{n-1}, y_{n}\right)$ in the plane $y_{n-1}-y_{n}$ when $(r-z) /(q-p)=1$.

Next, subtracting (95) from (94), we have

$$
z\left(J_{3}-J_{2}\right)+\left(q J_{2}+1\right)\left(J_{3}-J_{1}\right)+\left(J_{3}+p\right)\left(J_{1}-J_{2}\right)=0 .
$$

Finally, subtracting (96) from (97), we have

$$
z\left(J_{2}-J_{1}\right)+\left(q J_{1}+1\right)\left(J_{2}-J_{3}\right)+\left(J_{2}+p\right)\left(J_{3}-J_{1}\right)=0 .
$$

But, under the assumption $y_{n-3}>(r-z) /(q-p)$, $y_{n-2}<1$, whereas $1<y_{n-1}<(r-z) /(q-p)$, clearly

$$
J_{2}<J_{1}<J_{3} .
$$

As such, the left-hand side of (98) is negative, which is a contradiction.

(3) $(r-z) /(q-p)=1$. In this case, the dynamics of $\left(y_{n-1}, y_{n}\right)$ are depicted in Figure 12.

By Remark 11(2) we have the following cases.

(1) $\left(y_{n-1}, y_{n}\right) \in R_{1} \cup R_{2} \Rightarrow\left(y_{n}, y_{n+1}\right) \in R_{3}$.

(2) $\left(y_{n-1}, y_{n}\right) \in R_{3} \cup R_{4} \Rightarrow\left(y_{n}, y_{n+1}\right) \in R_{2}$.

By Lemma $14, \bar{y}=1$. Our interest now is to show that the pairs $\left(y_{n-1}, y_{n}\right)$ cannot stay forever in $R_{2} \cup R_{3}$.

Assume that $y_{2 n}<1$, whereas $y_{2 n-1}>1$ for all $n$. Then

$$
\begin{aligned}
y_{2 n+1}-y_{2 n-1} & <\frac{r+p y_{2 n}}{z+q y_{2 n}}-y_{2 n-1} \\
& <\frac{\left(r-z y_{2 n-1}\right)+\left(p-q y_{2 n-1}\right) y_{2 n}}{z+q y_{2 n}} \\
& <\frac{q y_{2 n-1}-p}{z+q y_{2 n}}\left[\frac{r-z y_{2 n-1}}{q y_{2 n-1}-p}-y_{2 n}\right] .
\end{aligned}
$$

Since $p<q, r>z$, and $y_{2 n-1}>1$ then $\left(r-z y_{2 n-1}\right) /\left(q y_{2 n-1}-\right.$ $p)<(r-z) /(q-p)$. Furthermore, $y_{2 n}<1=(r-z) /(q-p)$, so the solution is decreasing. With that in mind,

$$
\begin{aligned}
y_{2 n+1}-y_{2 n-1} & <\frac{q y_{2 n-1}-p}{z+q y_{2 n}}\left[\frac{r-z}{q-p}-y_{2 n}\right] \\
& <\frac{q(1)-p}{z+q((r-z) /(q-p))}\left[\frac{r-z}{q-p}-\frac{r-z}{q-p}\right] \\
& =0 .
\end{aligned}
$$

Thus the odd terms converge and so do the even terms. This implies the existence of a period-two solution which is a contradiction since, by Theorem 9, (6) does not possess a period-two solution.

The proof is complete.

4.2. Case 2: $p>q$. When $p>q$, Identities (49) and (50) are equivalent to the following:

$$
y_{n+1}-1=\frac{p-q}{z+q y_{n}+y_{n-1}}\left[y_{n}-\frac{z-r}{p-q}\right], \quad \text { for } n \geq 0 ;
$$

$$
y_{n+1}-\frac{p}{q}=\frac{p-q}{q\left(z+q y_{n}+y_{n-1}\right)}\left[\frac{q r-p z}{p-q}-y_{n-1}\right] \text {, }
$$

for $n \geq 0$.

The following remark is straightforward from Identities (102) and (103).

Remark 16. Let $\left\{y_{n}\right\}_{n=-1}^{\infty}$ be a positive solution of (6). Then the following statements are true when $p>q$.

(1) $y_{n+1}<1$ if and only if $y_{n}<(z-r) /(p-q)$.

(2) $y_{n+1}>p / q$ if and only if $y_{n-1}<(q r-p z) /(p-q)$.

Lemma 17. Assume that

$$
p>q
$$

Then we have two cases to be considered.

(1) $r \geq z$.

(a) If $(q r-p z) /(p-q) \leq p / q$ then $\bar{y} \leq p / q$.
(b) If $(q r-p z) /(p-q)>p / q$ then $\bar{y}>p / q$.

(2) $r<z$.

(a) If $(z-r) /(p-q) \leq 1$ then $\bar{y} \geq 1$.

(b) If $(z-r) /(p-q)>1$ then $\bar{y}<1$.

Proof. The proof is similar to the proof of Lemmas 12 and 14 and will be omitted. 
TABLE 2: Signs of $\partial f / \partial y_{n}$ and $\partial f / \partial y_{n-1}$ of (6) when $p>q$.

\begin{tabular}{|c|c|c|c|c|c|c|c|c|}
\hline Case & $q r-p z$ & $z-r$ & $\frac{\partial f\left(y_{n}, y_{n-1}\right)}{\partial y_{n}}=$ & $=\frac{p-q}{\left(z+q y_{n}+y_{n-1}\right)^{2}}$ & {$\left[y_{n-1}-\frac{q r-p z}{p-q}\right.$} & $\frac{\partial f\left(y_{n}, y_{n-1}\right)}{\partial y_{n-1}}=$ & $\frac{p-q}{\left(z+q y_{n}+y_{n-1}\right)^{2}}$ & $\frac{z-r}{p-q}-y_{n}$ \\
\hline 1 & + & - & + if $y_{n-1}>$ & $\frac{q r-p z}{p-q}$ and - if $y$ & ${ }_{n-1}<\frac{q r-p z}{p-q}$ & & - & \\
\hline 2 & 0 & - & & $\begin{array}{l}1 \\
+\end{array}$ & & & - & \\
\hline 3 & - & - & & + & & & - & \\
\hline 4 & - & 0 & & + & & & - & \\
\hline 5 & - & + & & + & & - if $y_{n}>$ & $\frac{z-r}{p-q}$ and + if $y_{n}<$ & $\frac{z-r}{p-q}$ \\
\hline
\end{tabular}

TABLE 3: Signs of $\partial f / \partial y_{n}$ and $\partial f / \partial y_{n-1}$ of (6) when $p=q$.

\begin{tabular}{lccc}
\hline Case & $z-r$ & $\frac{\partial f\left(y_{n}, y_{n-1}\right)}{\partial y_{n}}=\frac{q(z-r)}{\left(z+q y_{n}+y_{n-1}\right)^{2}}$ & $\frac{\partial f\left(y_{n}, y_{n-1}\right)}{\partial y_{n-1}}=\frac{z-r}{\left(z+q y_{n}+y_{n-1}\right)^{2}}$ \\
\hline 1 & + & + & + \\
2 & - & - & - \\
\hline
\end{tabular}

Table 2 gives the signs of $\partial f\left(y_{n}, y_{n-1}\right) / \partial y_{n}$ and $\partial f\left(y_{n}\right.$, $\left.y_{n-1}\right) / \partial y_{n-1}$ of (6) in all possible nondegenerate cases when $p>q$.

By Remark 16, Lemma 17, and Table 2, we obtain the following key result.

Theorem 18. Assume that

$$
p>q
$$

Then we have two cases to be considered.

(1) $r \geq z$.

(a) If $q r-p z \leq 0$, then $[1, p / q]$ is invariant.

(b) If $q r-p z>0$, then we have two subcases to be considered.

(i) If $(q r-p z) /(p-q) \leq p / q$, then every positive solution of (6) eventually enters and remains in the interval $[(q r-p z) /(p-q), p / q]$.

(ii) If $(q r-p z) /(p-q)>p / q$, then every positive solution of (6) eventually enters and remains in the interval $[p / q,(q r-p z) /(p-q)]$.

(2) $r<z$.

(a) $(z-r) /(p-q) \leq 1$, then every positive solution of (6) eventually enters and remains in the interval $[1, p / q]$.

(b) $(z-r) /(p-q)>1$, then every positive solution of (6) eventually enters and remains in the interval $(0,1)$.

Proof. (1) Assume that $p>q$ and $r \geq z$. Identity (102) shows that $y_{n}>1$ for all $n \geq 0$.

Here we have two cases to be considered.

(a) If $q r-p z \leq 0$.

In view of Table 2, Cases 2, 3, and 4, the function $f\left(y_{n-1}, y_{n}\right)$ is increasing in $y_{n}$ and decreasing in $y_{n-1}$ for all values of $y_{n}$ and $y_{n-1}$. Using the decreasing character of $f$ in $y_{n-1}$, and the increasing character in $y_{n}$, we obtain

$$
\begin{aligned}
1 & <\frac{q(r+1)+p^{2}}{q(z+1)+p q}=f\left(\frac{p}{q}, 1\right)<f\left(y_{n}, y_{n-1}\right) \\
& <f\left(1, \frac{p}{q}\right) \\
& =\frac{q(r+p)+p}{q(z+q)+p}<\frac{p}{q} .
\end{aligned}
$$

(b) If $q r-p z>0$.

In this case, the plane $y_{n-1}-y_{n}$ divides into the regions depicted in Figure 13.

We have to treat the cases $(q r-p z) /(p-q)<p / q$, $(q r-p z) /(p-q)>p / q$, and $(q r-p z) /(p-q)=p / q$.

The proof is similar to that of Theorem 15 and will be omitted.

(2) Assume that $p>q$ and $r<z$. Identity (103) shows that $y_{n}<p / q$ for all $n \geq 0$. In this case, the square $[0, p / q] \times$ $[0, p / q]$ divides into the regions depicted in Figure 14 .

We have to treat the cases $(z-r) /(p-q)<1,(z-r) /(p-$ $q)>1$, and $(z-r) /(p-q)=1$.

The proof is similar to that of Theorem 13, part 2, and will be omitted.

The proof is complete.

4.3. Case 3: $p=q$. When $p=q$, Identities (49) and (50) reduce to the following:

$$
y_{n+1}-1=\frac{r-z}{z+q y_{n}+y_{n-1}}, \quad \text { for } n \geq 0 .
$$

Table 3 gives the signs of $\partial f / \partial x$ and $\partial f / \partial y$ in all possible nondegenerate cases when $p=q$.

By Identity (107) and Table 3, we obtain the following result. 


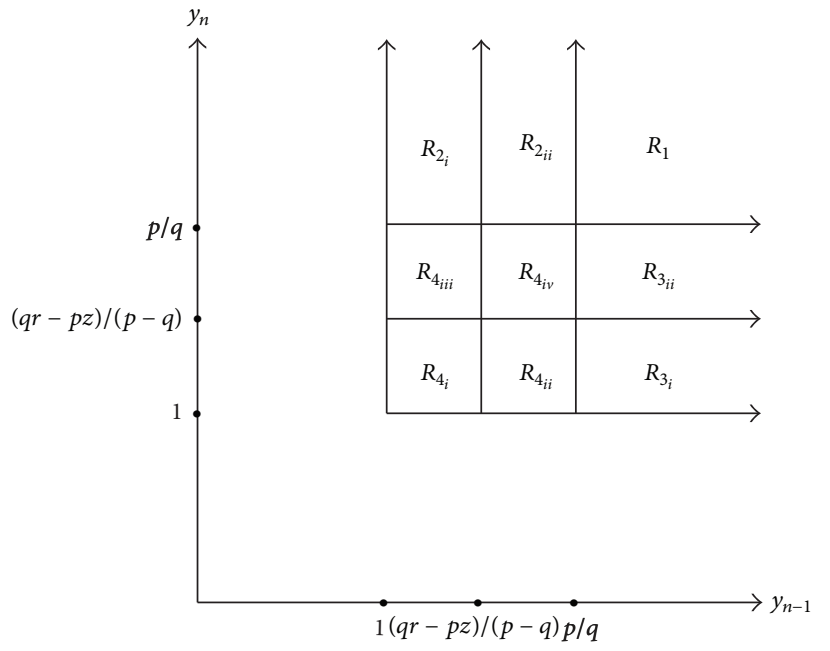

(a)

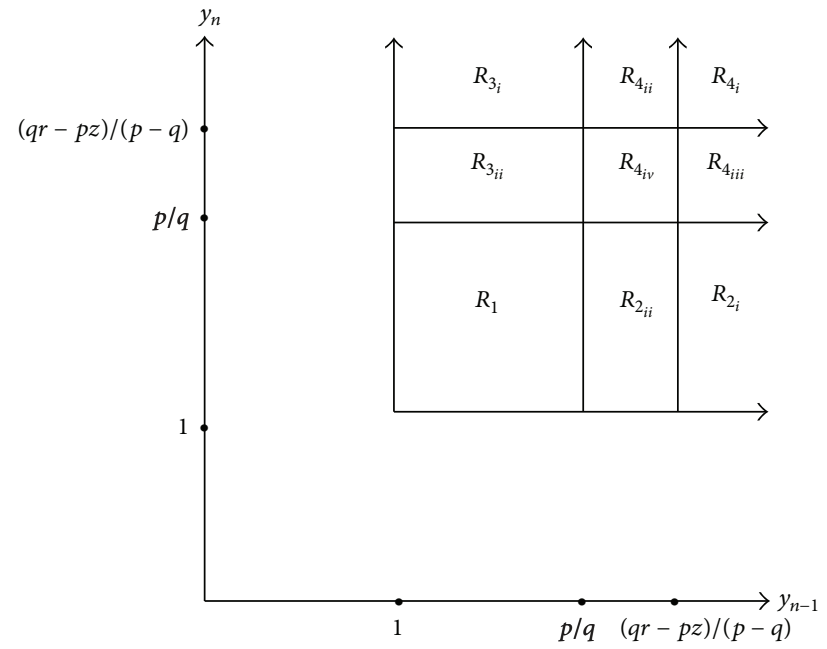

(b)

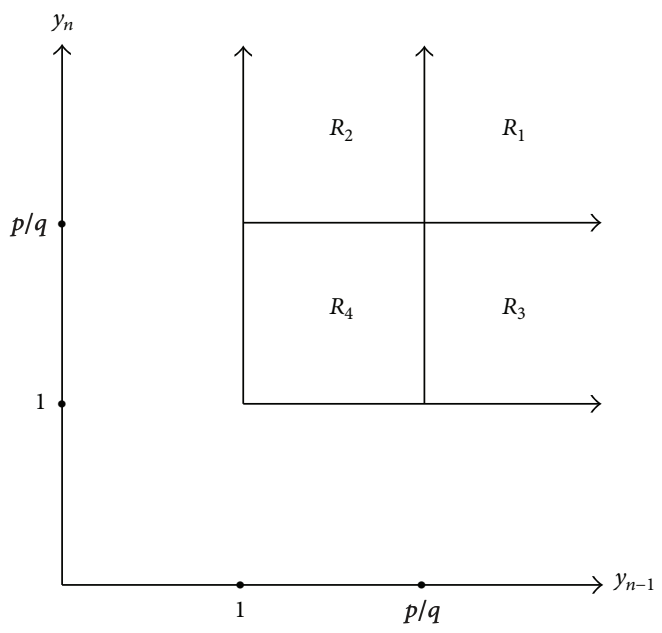

(c)

FIGURE 13: All possible regions in the plane $y_{n-1}-y_{n}$ when $p>q, r \geq z$, and $q r-p z>0 ;($ a) $(q r-p z) /(p-q)<p / q ;(\mathrm{b})(q r-p z) /(p-q)>p / q$; (c) $(q r-p z) /(p-q)=p / q$.

Theorem 19. When

$$
p=q
$$

equation (6) possesses the following invariant intervals.

(1) $[r / z, 1]$ if $r \leq z$.

(2) $(1, r / z)$ if $r>z$.

Proof. (1) Identity (107) shows that if $p=q$ and $r \leq z$ then $y_{n} \leq 1$ for all $n \geq 0$.

Notice that when $p=q$ and $r=z$ then the function $f(x, y)=1$ for all values of $x$ and $y$, while if $p=q$ and $r<z$ then by Table 3, Case 1 , the function $f(x, y)$ is increasing in both arguments for all values of $x$ and $y$. Using the increasing character of $f$ we have,

$$
\frac{r}{z}=f(0,0)<f(x, y)<f(1,1)=\frac{r+q+1}{z+q+1}<1 .
$$

(2) Identity (107) shows that if $p=q$ and $r>z$ then $y_{n}>1$ for all $n \geq 0$.

Furthermore, by Table 3, Case 2, the function $f(x, y)$ is decreasing in both arguments for all values of $x$ and $y$. Using the decreasing character of $f$ we have,

$$
1<\frac{r+p x+y}{z+p x+y}=f(x, y)<f(1,1)=\frac{r+q+1}{z+q+1}<\frac{r}{z} .
$$

The proof is complete.

Table 4 gives a summary of Theorems 13, 15, 18, and 19.

\section{Global Stability of Hyperbolic Equilibrium Solution}

The results about the global stability for the positive equilibrium of (6) are given in the following theorem. 


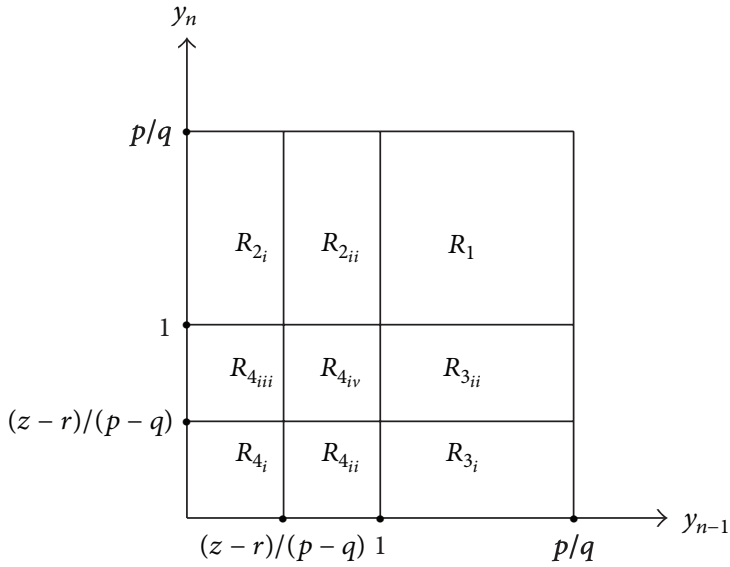

(a)

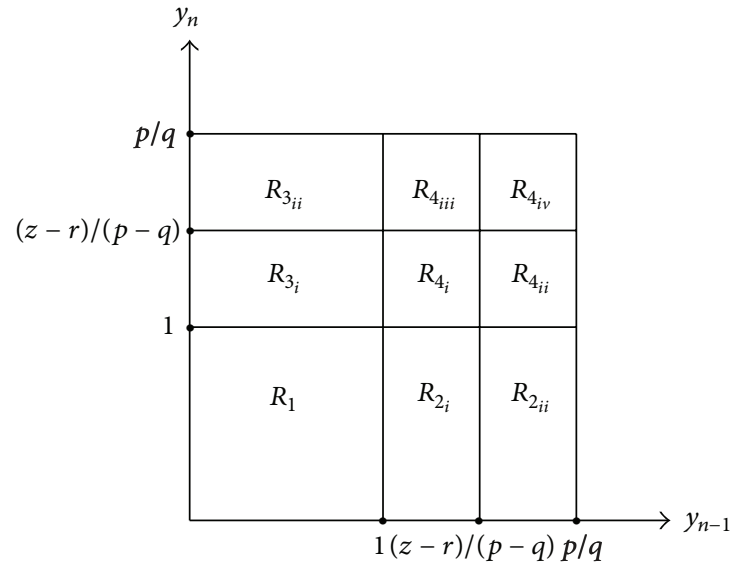

(b)

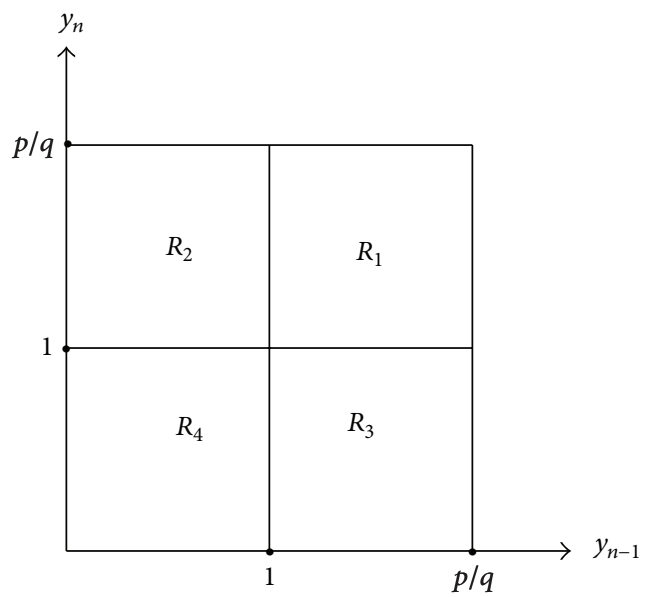

(c)

FIGURE 14: All possible regions in the plane $y_{n-1}-y_{n}$ when $p>q$ and $r<z$; (a) $(z-r) /(p-q)<1 ;(\mathrm{b})(z-r) /(p-q)>1 ;(\mathrm{c})(z-r) /(p-q)=1$.

Theorem 20. (i) Assume that

$$
p+z \geq 1
$$

then the positive equilibrium of (6) is globally asymptotically stable.

(ii) Assume that

$$
p+z<1
$$

then the positive equilibrium of (6) is globally asymptotically stable if and only if

$$
r>\frac{(1-p-z)[q(1-p-z)-(1+3 p-z)]}{4} .
$$

Proof. We have established the local stability of the equilibrium solution in Theorem 10. To complete the proof it remains to show that the equilibrium $\bar{y}$ is a global attractor. Let

$$
f(x, y)=\frac{r+p x+y}{z+q x+y} .
$$

We consider the following three cases.
Case 1: $p<q$. It follows from Table 4 that each of $(0, p / q)$, $[p / q, 1],[(r-z) /(q-p), 1]$, and $[1,(r-z) /(q-p)]$ is an invariant interval for (6) according to special conditions. Furthermore, we have the following.

(a) The function (114) in $(0, p / q)$ is increasing in both arguments. Now the conclusion of Theorem 20 follows as consequence of Theorem 8 .

(b) The function (114) in $[p / q, 1]$ and $[(r-z) /(q-p), 1]$ is nonincreasing in $x$ and nondecreasing in $y$. Also, when (111) or (112) and (113) hold, (6) has no prime period-two solution as we showed in Theorem 9. Now the result is a consequence of Theorem 5 .

(c) The function (114) in $[1,(r-z) /(q-p)]$ is decreasing in both arguments. In order to employ Theorem 7 , we have to show that the only solution of the system

$$
M=\frac{r+p m+m}{z+q m+m}, \quad m=\frac{r+p M+M}{z+q M+M}
$$


TABLE 4: Summary of Theorems 13, 15, 18, and 19.

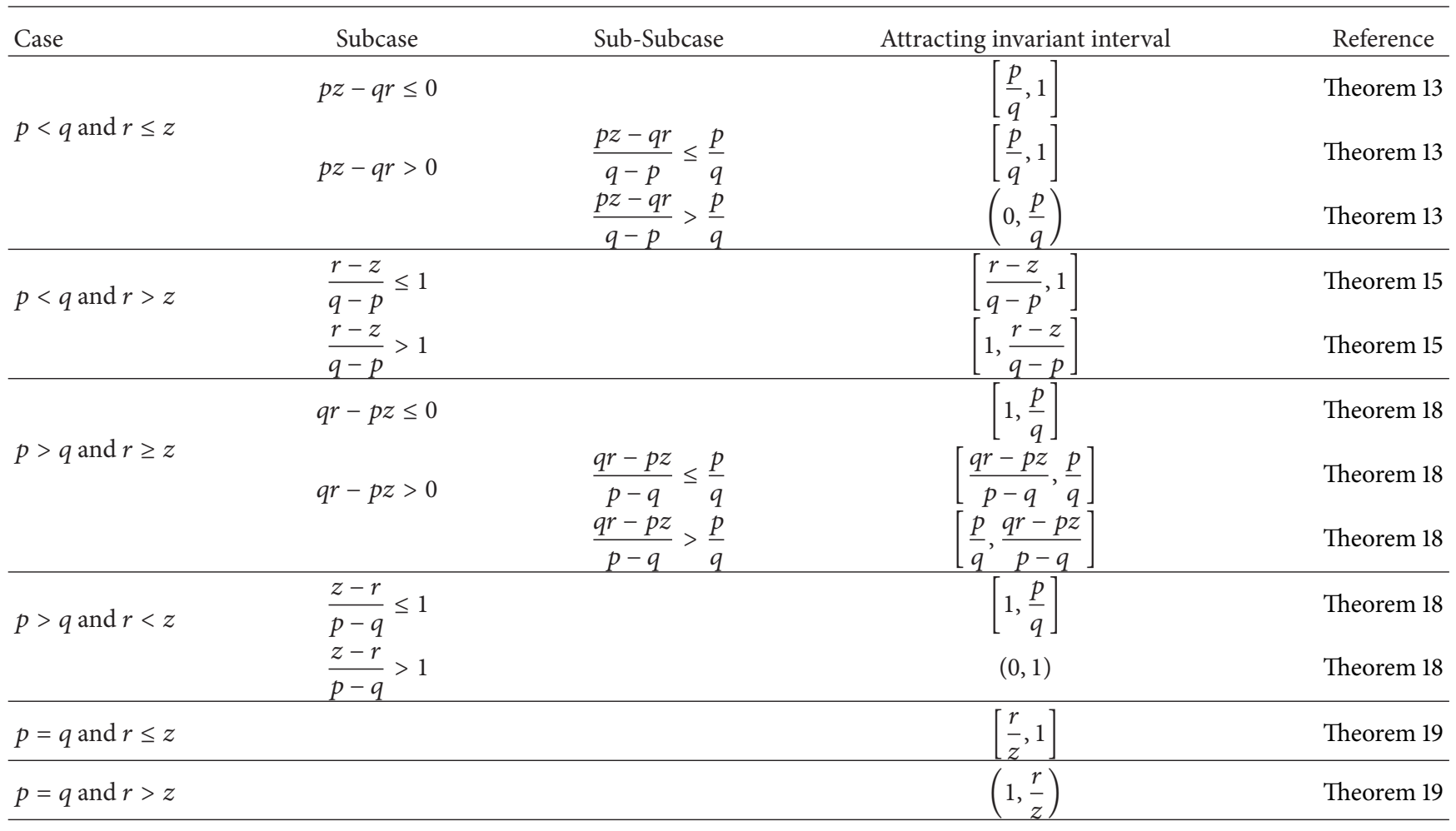

is $M=m$. This system is equivalent to

$$
(M-m)(1+p+z)=0
$$

which implies $M=m$.

Now the result is a consequence of Theorem 7 .

Case 2: $p>q$. It follows from Table 4 that each of $(0,1)$, $[1, p / q],[(q r-p z) /(p-q), p / q]$, and $(p / q,(q r-p z) /(p-q))$ is an invariant interval for (6) according to special conditions. Furthermore, we have the following.

(a) The function $(114)$ in $(0,1)$ is increasing in both arguments. Now the conclusion of Theorem 20 follows as consequence of Theorems 8.

(b) The function (114) in $[1, p / q]$ and $[(q r-p z) /(p-$ $q), p / q$ ] is nondecreasing in $x$ and nonincreasing in $y$. In order to employ Theorem 6, we have to show that the only solution of the system

$$
M=\frac{r+p M+m}{z+q M+m}, \quad m=\frac{r+p m+M}{z+q m+M}
$$

is $M=m$. This system is equivalent to

$$
(M-m)(1-p+z+q(M+m))=0
$$

which implies $M=m$.

Now the result is a consequence of Theorem 6. (c) The function (114) in $(p / q,(q r-p z) /(p-q))$ is decreasing in both arguments. Also, the only solution of the system

$$
M=\frac{r+p m+m}{z+q m+m}, \quad m=\frac{r+p M+M}{z+q M+M}
$$

is $M=m$. Now the result is a consequence of Theorem 7 .

Case 3: $p=q$. It follows from Table 4 that each of $[r / z, 1]$ and $(1, r / z)$ is an invariant interval for (6) according to special conditions. Furthermore, we have the following.

(a) The function (114) in $[r / z, 1]$ is nondecreasing in both arguments. Now the conclusion of Theorem 20 follows as consequence of Theorem 8 .

(b) The function (114) in $(1, r / z)$ is decreasing in both arguments. Also, the only solution of the system

$$
M=\frac{r+p m+m}{z+p m+m}, \quad m=\frac{r+p M+M}{z+p M+M}
$$

is $M=m$. Now the result is a consequence of Theorem 7.

The proof is complete.

\section{Numerical Examples}

In order to illustrate the results of the previous sections and to support our theoretical discussion, we consider several numerical examples generated by MATLAB. 


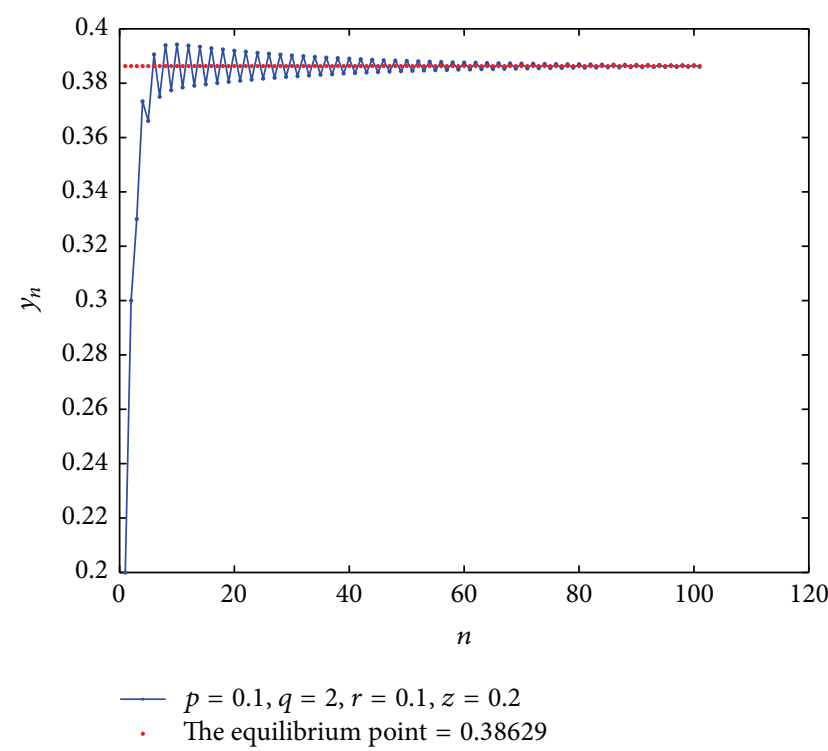

FIGURE 15: Dynamics of $y_{n+1}=\left(0.1+0.1 y_{n}+y_{n-1}\right) /\left(0.2+2 y_{n}+y_{n-1}\right)$.

Example 1. Consider the following equation:

$$
y_{n+1}=\frac{0.1+0.1 y_{n}+y_{n-1}}{0.2+2 y_{n}+y_{n-1}} .
$$

Since $r=0.1$ satisfies condition (39), by Theorem 10, the equilibrium is locally asymptotically stable. Indeed, $r \leq z$ and $p z-q r \leq 0$; Theorem 13 implies that every positive solution of (121) eventually enters and remains in the interval $[p / q, 1]$. Furthermore, the equilibrium $\bar{y}$ is globally asymptotically stable by Theorem 20. The dynamics of (121) are shown in Figure 15.

Example 2. Consider the following equation:

$$
y_{n+1}=\frac{0.001+0.01 y_{n}+y_{n-1}}{0.9+1.5 y_{n}+y_{n-1}} .
$$

Since $r=0.001$ satisfies condition (39), by Theorem 10, the equilibrium is locally asymptotically stable. Indeed, $r<z$, $p z-q r>0$, and $(p z-q r) /(q-p)<p / q$; Theorem 13 implies that every positive solution of (122) eventually enters and remains in the interval $[p / q, 1]$. Furthermore, the equilibrium $\bar{y}$ is globally asymptotically stable by Theorem 20 . The dynamics of (122) are shown in Figure 16.

Example 3. Consider the following equation:

$$
y_{n+1}=\frac{8+2 y_{n}+y_{n-1}}{4+0.5 y_{n}+y_{n-1}} .
$$

Since condition (37) is satisfied, by Theorem 10, the equilibrium is locally asymptotically stable. Indeed, $p>q, r \geq z$, and $q r-p z \leq 0$; Theorem 18 implies that every positive solution of (123) eventually enters and remains in the interval [1, $p / q]$. Furthermore, the equilibrium $\bar{y}$ is globally asymptotically stable by Theorem 20 . The dynamics of (123) are shown in Figure 17.

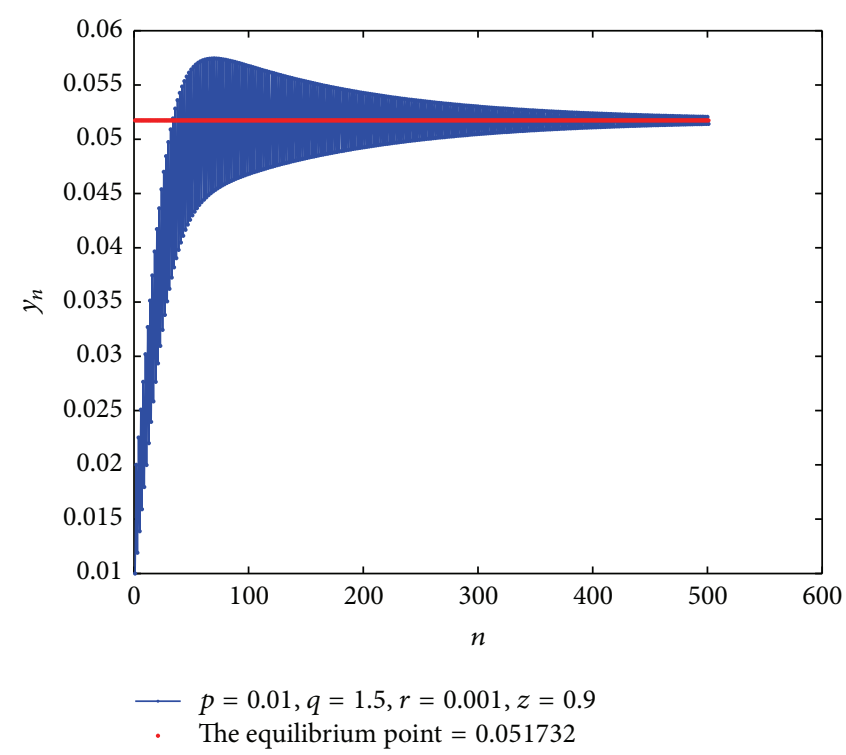

FIGURE 16: Dynamics of $y_{n+1}=\left(0.001+0.01 y_{n}+y_{n-1}\right) /\left(0.9+1.5 y_{n}+\right.$ $\left.y_{n-1}\right)$.

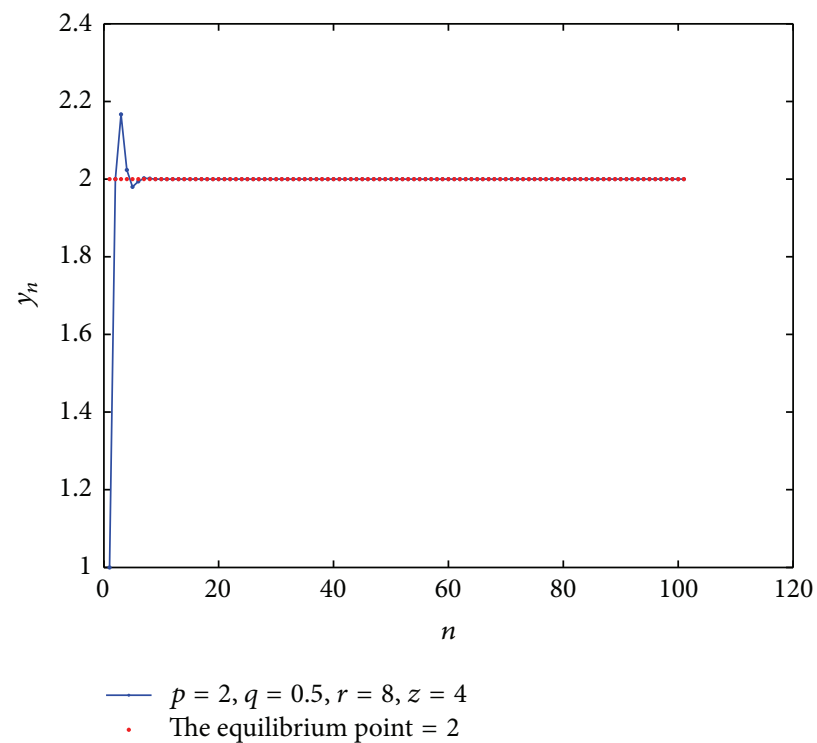

FIGURE 17: Dynamics of $y_{n+1}=\left(8+2 y_{n}+y_{n-1}\right) /\left(4+0.5 y_{n}+y_{n-1}\right)$.

Example 4. Consider the following equation:

$$
y_{n+1}=\frac{10+2 y_{n}+y_{n-1}}{0.3+0.6 y_{n}+y_{n-1}} \text {. }
$$

Since condition (37), by Theorem 10, the equilibrium is locally asymptotically stable. Indeed, $r>z, q r-p z>0$, and ( $q r-$ $p z) /(p-q)>p / q$; Theorem 18 implies that every positive solution of (124) eventually enters and remains in the interval $[p / q,(q r-p z) /(p-q)]$. Furthermore, the equilibrium $\bar{y}$ is globally asymptotically stable by Theorem 20 . The dynamics of (124) are shown in Figure 18. 


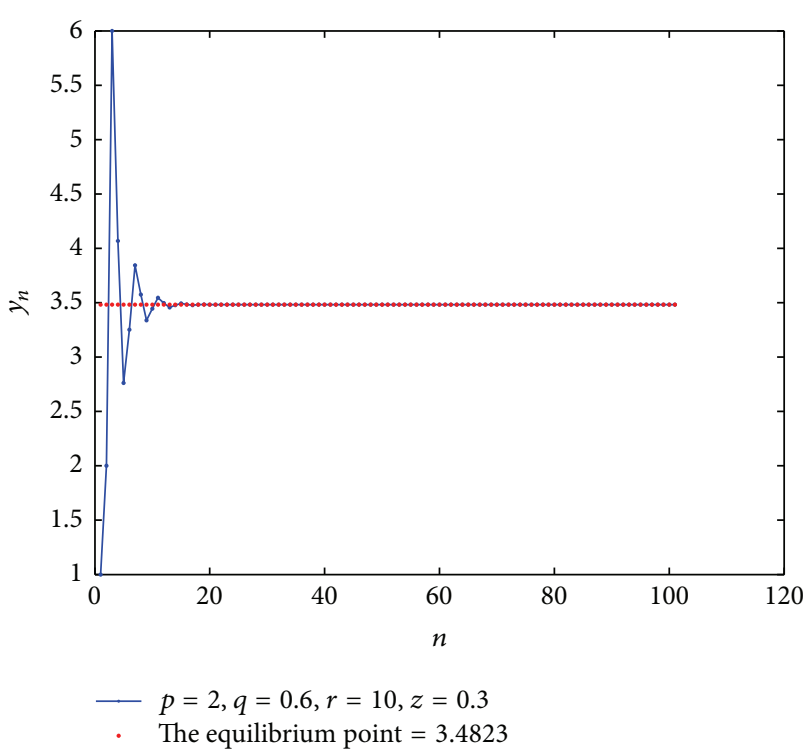

FIGURE 18: Dynamics of $y_{n+1}=\left(10+2 y_{n}+y_{n-1}\right) /\left(0.3+0.6 y_{n}+y_{n-1}\right)$.

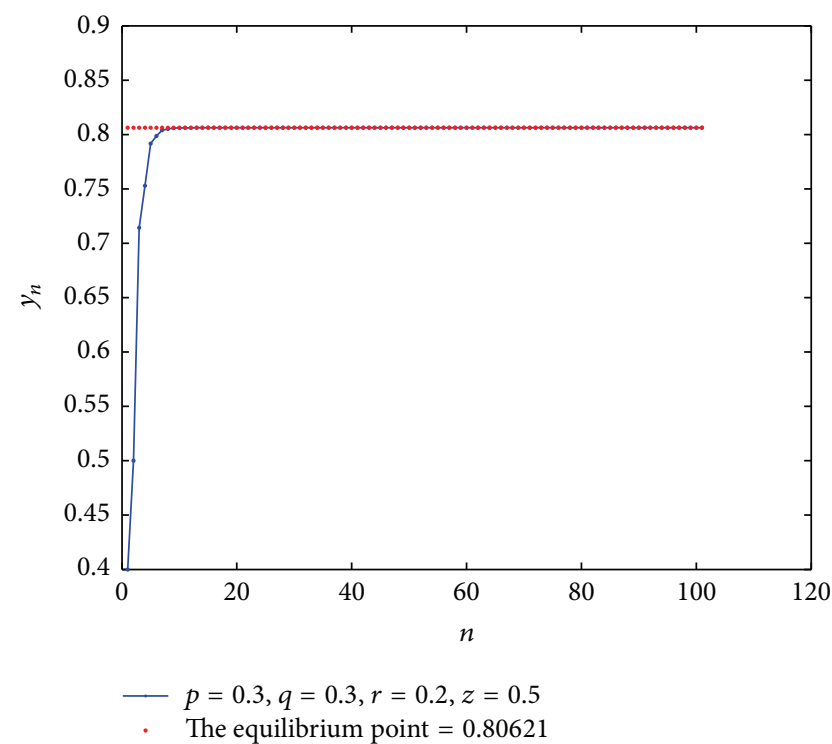

FIGURE 19: Dynamics of $y_{n+1}=\left(0.2+0.3 y_{n}+y_{n-1}\right) /\left(0.5+0.3 y_{n}+y_{n-1}\right)$.

Example 5. Consider the following example:

$$
y_{n+1}=\frac{0.2+0.3 y_{n}+y_{n-1}}{0.5+0.3 y_{n}+y_{n-1}}
$$

Since $r=0.02$ satisfies condition (39), by Theorem 10, the equilibrium is locally asymptotically stable. Indeed, $p=q$ and $r \leq z$; Theorem 19 implies that every positive solution of (125) eventually enters and remains in the interval $[r / z, 1]$. Furthermore, the equilibrium $\bar{y}$ is globally asymptotically stable by Theorem 20 . The dynamics of (125) are shown in Figure 19.

\section{Conclusion}

In this paper, we have established the global stability of the hyperbolic equilibrium solutions of the second order rational difference equation

$$
x_{n+1}=\frac{\alpha+\beta x_{n}+\gamma x_{n-1}}{A+B x_{n}+C x_{n-1}}, \quad n=0,1,2, \ldots,
$$

where the parameters $\alpha, \beta, \gamma, A, B, C$ are positive real numbers and the initial conditions $x_{-1}, x_{0}$ are nonnegative real numbers. Particularly, we showed that

$$
\text { Local Asymptotic Stability } \Longrightarrow \text { Global Attractivity. }
$$

However, it is natural to ask about the global stability of the nonhyperbolic equilibrium solutions for the equation of the form mentioned above. In particular, one may want to investigate the necessary and sufficient conditions for the equation to have nonhyperbolic solution and completely examine the existence of intervals which attract all solutions of the equation in order to obtain a convergence result for the the nonhyperbolic equilibrium solutions of the equation of the form mentioned above.

We consider the aforementioned result as a step forward in investigating bigger classes of difference equations which afford the LGAS property; that is, local stability of an equilibrium implies its global stability.

\section{Conflict of Interests}

The authors declare that there is no conflict of interests regarding the publication of this paper.

\section{Acknowledgments}

This paper is dedicated to Gerry Ladas on the occasion of his retirement. The authors are very grateful to the anonymous referees for carefully reading the paper and for their comments and valuable suggestions that lead to an improvement in the paper.

\section{References}

[1] R. Abu-Saris, C. Çina, and I. Yalçinkaya, "On the asymptotic stability of $x_{n+1}=\left(a+x_{n} x_{n-k}\right) /\left(x_{n}+x_{n-k}\right)$," Computers \& Mathematics with Applications, vol. 56, no. 5, pp. 1172-1175, 2008.

[2] R. P. Agarwal and E. M. Elsayed, "Periodicity and stability of solutions of higher order rational difference equation," Advanced Studies in Contemporary Mathematics, vol. 17, no. 2, pp. 181-201, 2008.

[3] Y. S. Huang and P. M. Knopf, "Boundedness of positive solutions of second-order rational difference equations," Journal of Difference Equations and Applications, vol. 10, no. 11, pp. 935940, 2004.

[4] Y. S. Huang and P. M. Knopf, "Boundedness and some convergence properties of the difference equation," Journal of Difference Equations and Applications, vol. 18, no. 1, pp. 27-55, 2012. 
[5] A. Raouf, "Global behaviour of the rational Riccati difference equation of order two: the general case," Journal of Difference Equations and Applications, vol. 18, no. 6, pp. 947-961, 2012.

[6] Q. Shi, Q. Xiao, G. Yuan, and X. Liu, "Dynamic behavior of a nonlinear rational difference equation and generalization," Advances in Difference Equations, vol. 2011, article 36, 2011.

[7] E. M. E. Zayed and M. A. El-Moneam, "On the rational recursive sequence," Communications on Applied Nonlinear Analysis, vol. 15, no. 2, pp. 47-57, 2008.

[8] E. M. E. Zayed and M. A. El-Moneam, "On the global asymptotic stability for a rational recursive sequence," Iranian Journal of Science and Technology, Transaction A: Science, vol. 35, no. 4, pp. 333-339, 2011.

[9] E. M. E. Zayed, A. B. Shamardan, and T. A. Nofal, "On the rational recursive sequence $x_{n+1}=\left(\alpha-\beta x_{n}\right) /\left(\gamma-\delta x_{n}-\right.$ $\left.x_{n-k}\right)$," International Journal of Mathematics and Mathematical Sciences, vol. 2008, Article ID 391265, 15 pages, 2008.

[10] R. P. Agarwal, Difference Equations and Inequalities: Theory, Methods, and Applications, Marcel Dekker, New York, NY, USA, 2000.

[11] S. N. Elaydi, An Introduction to Difference Equations, Springer, New York, NY, USA, 2005.

[12] W. G. Kelley and A. C. Peterson, Difference Equations: An Introduction with Applications, Harcour Academic Press, New York, NY, USA, 2001.

[13] V. L. Kocic and G. Ladas, Global Behavior of Nonlinear Difference Equations of Higher Order with Application, vol. 256 of Mathematics and its Applications, Kluwer Academic, Dordrecht, The Netherlands, 1993.

[14] E. Camouzis and G. Ladas, Dynamics of Third-Order Rational Difference Equationsn with Open Problem and Conjectures, Chapman \& Hall/CRC, Boca Raton, Fla, USA, 2008.

[15] M. R. S. Kulenović and G. Ladas, Dynamics of Second-Order Rational Difference Equations with Open Problem and Conjectures, Chapman \& Hall/CRC, Boca Raton, Fla, USA, 2002.

[16] A. M. Amleh, E. A. Grove, G. Ladas, and D. A. Georgiou, "On the recursive sequence $x_{n+1}=a+x_{n-1} / x_{n}$ ", Journal of Mathematical Analysis and Applications, vol. 233, no. 2, pp. 790798, 1999.

[17] K. Cunningham, M. R. S. Kulenović, G. Ladas, and S. V. Valicenti, "On the recursive sequence $x_{n+1}=\left(\alpha+\beta x_{n-1}\right) /\left(b x_{n}+\right.$ $\left(x_{n-1}\right)$," Nonlinear Analysis: Theory, Methods \& Applications, vol. 47, no. 7, pp. 4603-4614, 2001.

[18] C. Gibbons, M. R. S. Kulenovic, and G. Ladas, "On the recursive sequence $x_{n+1}=\left(\alpha+\beta x_{n-1}\right) /\left(\gamma+x_{n}\right)$," Mathematical Sciences Research Hotline, vol. 4, no. 2, pp. 1-11, 2000.

[19] C. H. Gibbons, M. R. S. Kulenovic, G. Ladas, and H. D. Voulov, "On the trichotomy character of $x_{n+1}=\left(\alpha+\beta x_{n}+\gamma x_{n-1}\right) /(\alpha+$ $\left.x_{n}\right)$," Journal of Difference Equations and Applications, vol. 8, no. 1, pp. 75-92, 2002.

[20] W. A. Kosmala, M. R. S. Kulenovic, G. Ladas, and C. T. Teixeira, "On the recursive sequence $y_{n+1}=\left(p+y_{n-1}\right) /\left(q y_{n}+y_{n-1}\right)$," Journal of Mathematical Analysis and Applications, vol. 251, no. 2, pp. 571-586, 2000.

[21] M. R. S. Kulenovic and G. Ladas, "Open problems and conjectures: on period two solutions of $x_{n+1}=\left(\alpha+\beta x_{n}+\gamma x_{n-1}\right) /(\alpha+$ $\left.b x_{n}+c x_{n-1}\right)$," Journal of Difference Equations and Applications, vol. 6, no. 5, pp. 641-646, 2000.

[22] M. R. Kulenovic, G. Ladas, and W. S. Sizer, "On the recursive sequence $x_{n+1}=\left(\alpha x_{n}+\beta x_{n-1}\right) /\left(\gamma x_{n}+c x_{n-1}\right)$, , Mathematical Sciences Research Hot-Line, vol. 2, no. 5, pp. 1-16, 1998.
[23] M. R. S. Kulenovic, G. Ladas, and N. R. Prokup, "On the recursive sequence $x_{n+1}=\left(\alpha x_{n}+\beta x_{n-1}\right)=\left(1+x_{n}\right)$," Journal of Difference Equations and Applications, vol. 6, no. 5, pp. 563$576,2000$.

[24] M. Saleh and S. Abu-Baha, "Dynamics of a higher order rational difference equation," Applied Mathematics and Computation, vol. 181, no. 1, pp. 84-102, 2006.

[25] T. Sun and H. Xi, "Global asymptotic stability of a higher order rational difference equation," Journal of Mathematical Analysis and Applications, vol. 330, no. 1, pp. 462-466, 2007.

[26] H. Xi and T. Sun, "Global behavior of a higher-order rational difference equation," Advances in Difference Equations, vol. 2006, Article ID 27637, 7 pages, 2006.

[27] X.-X. Yan, W.-T. Li, and Z. Zhao, "Global asymptotic stability for a higher order nonlinear rational difference equations," Applied Mathematics and Computation, vol. 182, no. 2, pp. 1819-1831, 2006.

[28] D. C. Zhang, B. Shi, S. R. Yang, and M. J. Gai, "On the periodicity of two rational recursive sequences," Indian Journal of Pure and Applied Mathematics, vol. 34, no. 4, pp. 631-649, 2003.

[29] A. M. Amleh, E. Camouzis, and G. Ladas, "On secondorder rational difference equation. Part I," Journal of Difference Equations and Applications, vol. 13, no. 11, pp. 969-1004, 2007.

[30] A. M. Amleh, E. Camouzis, and G. Ladas, "On second-order rational difference equations, Part 2," Journal of Difference Equations and Applications, vol. 14, no. 2, pp. 215-228, 2008.

[31] S. E. Daş, "Dynamics of a nonlinear rational difference equation," Hacettepe Journal of Mathematics and Statistics, vol. 42, no. 1, pp. 9-14, 2013.

[32] M. Dehghan and R. Mazrooei-Sebdani, "Some characteristics of solutions of a class of rational difference equations," Kybernetes, vol. 37, no. 6, pp. 786-796, 2008.

[33] L.-X. Hu, W.-S. He, and H.-M. Xia, "Global asymptotic behavior of a rational difference equation," Applied Mathematics and Computation, vol. 218, no. 15, pp. 7818-7828, 2012.

[34] R. Karatas, "Global behavior of a higher order difference equation," Computers \& Mathematics with Applications, vol. 60, no. 3, pp. 830-839, 2010.

[35] D. Li, S. Zou, and M. Liao, "On a class of second-order nonlinear difference equation," Advances in Difference Equations, vol. 2011, article 46, 2011.

[36] G.-M. Tang, L.-X. Hu, and G. Ma, "Global stability of a rational difference equation," Discrete Dynamics in Nature and Society, vol. 2010, Article ID 432379, 17 pages, 2010.

[37] N. Touafek, "On a second order rational difference equation," Hacettepe Journal of Mathematics and Statistics, vol. 41, no. 6, pp. 867-874, 2012.

[38] Y. Wang, Q. Tu, Q. Wang, and C. Hu, "Dynamics of a high-order rational difference equation," Utilitas Mathematica, vol. 88, pp. 13-25, 2012.

[39] Q. Xiao and Q.-H. Shi, "Qualitative behavior of a rational difference equation $y_{n+1}=\left(y_{n}+y_{n-1}\right) /\left(p+y_{n} y_{n-1}\right)$," Advances in Difference Equations, vol. 2011, article 6, 2011.

[40] S. Atawna, R. Abu-Saris, and I. Hashim, "Local stability of period two cycles of second order rational difference equation," Discrete Dynamics in Nature and Society, vol. 2012, Article ID 969813, 11 pages, 2012. 


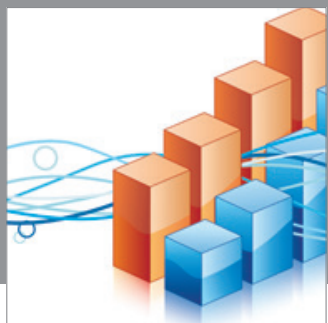

Advances in

Operations Research

mansans

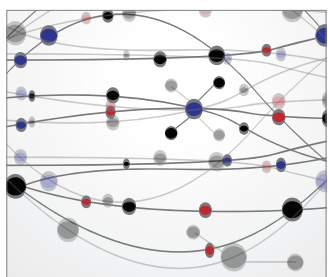

The Scientific World Journal
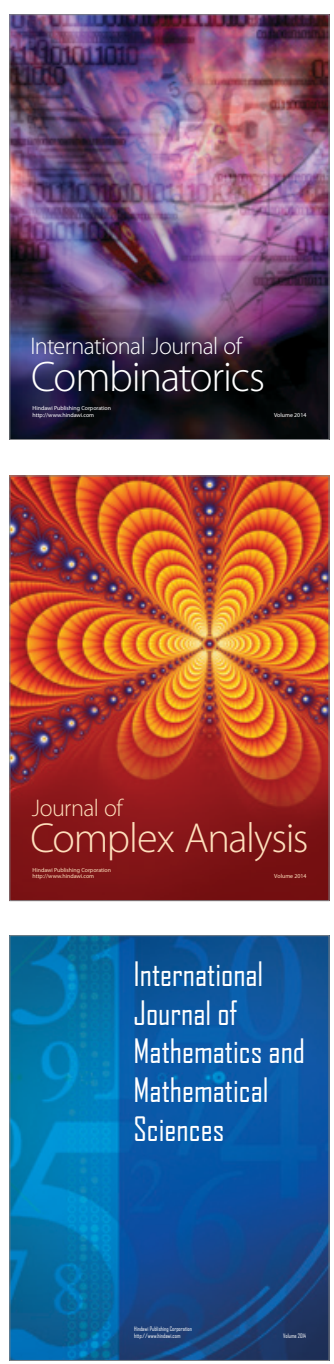
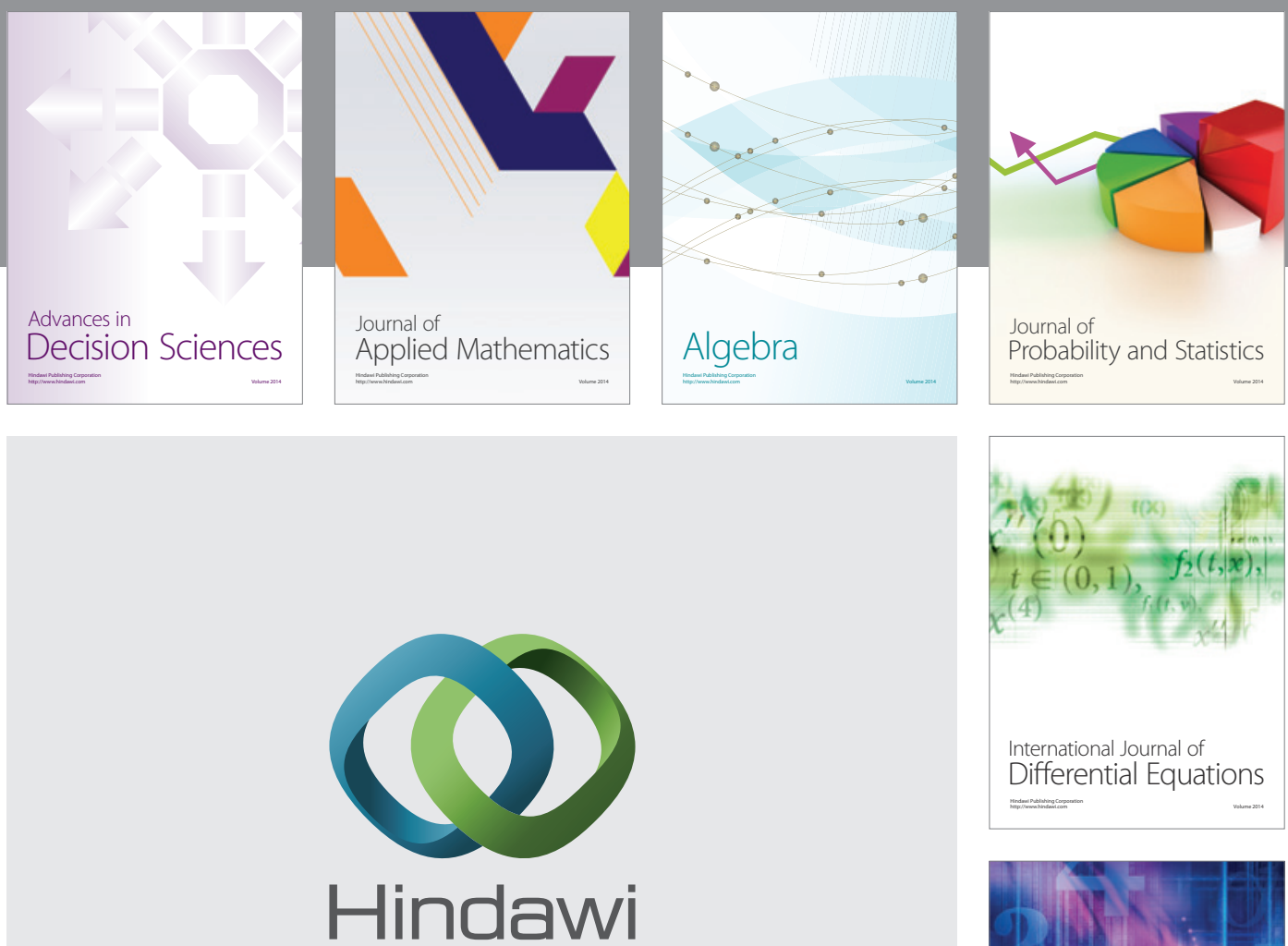

Submit your manuscripts at http://www.hindawi.com
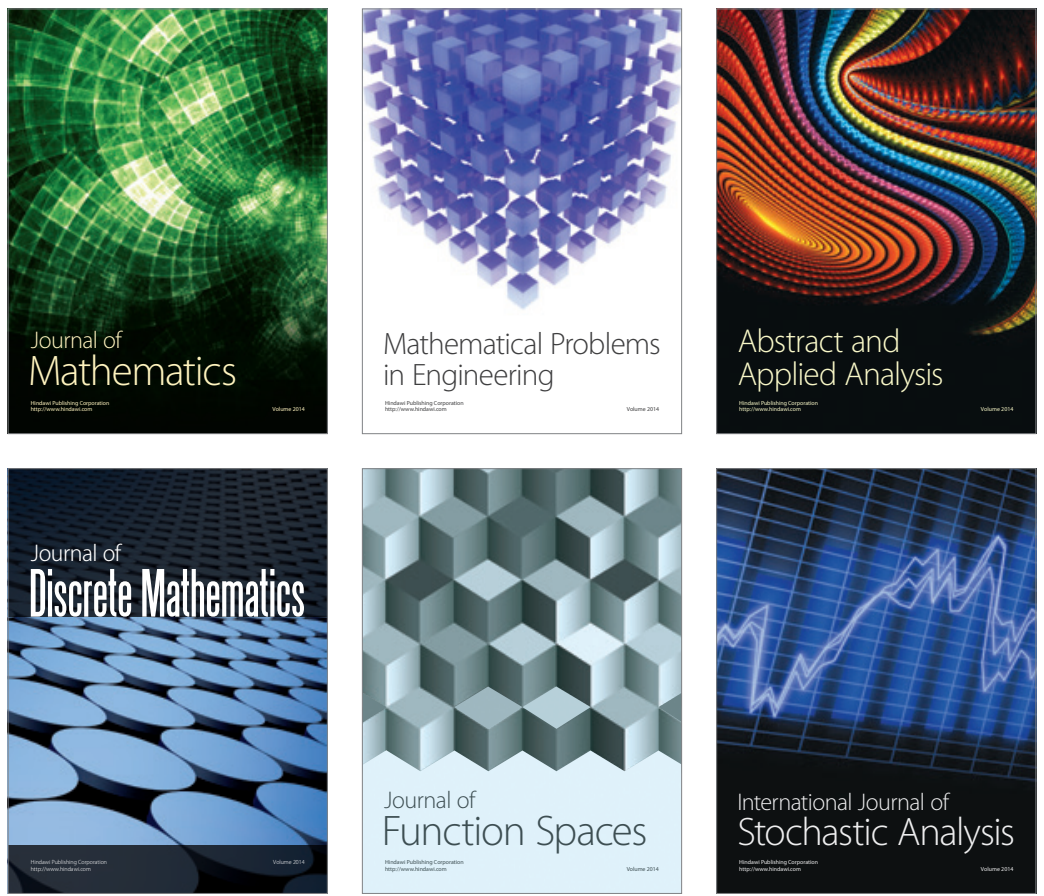

Journal of

Function Spaces

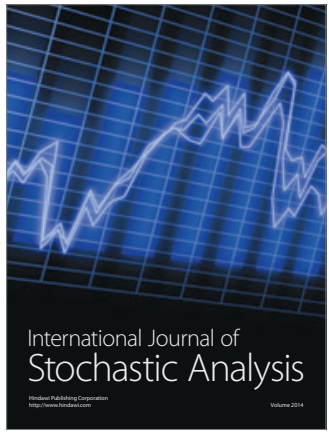

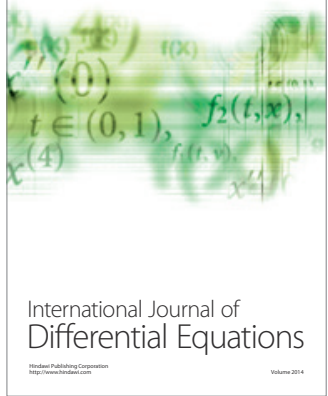
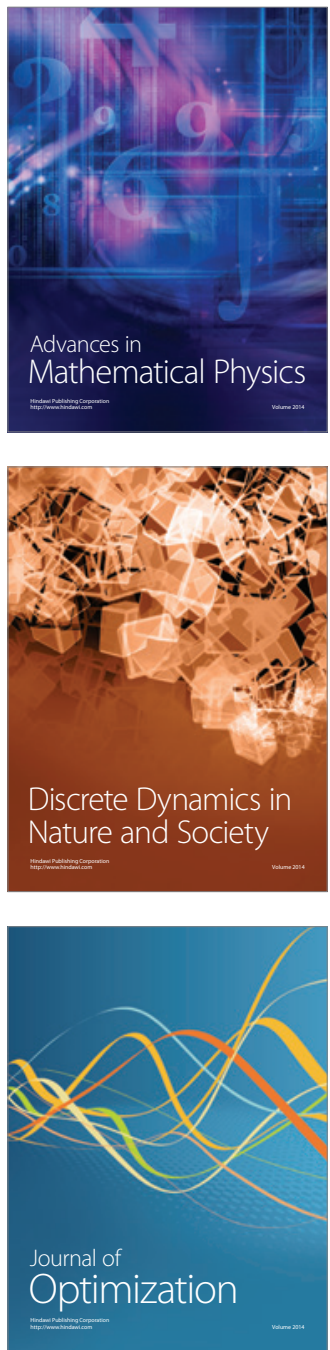